\title{
Halobacterium salinarum virus Chaos9, a Novel Halovirus Related to PhiH1 and PhiCh1
}

\author{
Mike Dyall-Smith ${ }^{1,2}{ }^{1}$, Peter Palm ${ }^{1}$, Gerhard Wanner ${ }^{3}$, Angela Witte ${ }^{4}$, Dieter Oesterhelt ${ }^{1}$ and \\ Friedhelm Pfeiffer $1, *$ iD \\ 1 Computational Biology Group, Max-Planck-Institute of Biochemistry, Am Klopferspitz 18, 82152 \\ Martinsried, Germany; mike.dyallsmith@gmail.com (M.D.-S.); hp.palm@kabelmail.de (P.P.); \\ oesterhe@biochem.mpg.de (D.O.) \\ 2 Veterinary Biosciences, Faculty of Veterinary and Agricultural Sciences, University of Melbourne, Parkville, \\ VIC 3052, Australia \\ 3 AG Ultrastrukturforschung, Biozentrum der LMU, Großhadernerstrasse 2-4, 82152 Martinsried, Germany; \\ wanner@1rz.uni-muenchen.de \\ 4 Department of Microbiology, Immunobiology and Genetics, MFPL Laboratories, University of Vienna, Dr. \\ Bohr-Gasse 9, 1030 Vienna, Austria; angela.witte@univie.ac.at \\ * Correspondence: fpf@biochem.mpg.de; Tel.: +49-89-8578-2323
}

Received: 29 January 2019; Accepted: 25 February 2019; Published: 1 March 2019

check for updates

\begin{abstract}
The unexpected lysis of a large culture of Halobacterium salinarum strain S9 was found to be caused by a novel myovirus, designated ChaoS9. Virus purification from the culture lysate revealed a homogeneous population of caudovirus-like particles. The viral genome is linear, dsDNA that is partially redundant and circularly permuted, has a unit length of 55,145 nt, a $\mathrm{G}+\mathrm{C} \%$ of 65.3, and has 85 predicted coding sequences (CDS) and one tRNA (Arg) gene. The left arm of the genome (0-28 kbp) encodes proteins similar in sequence to those from known caudoviruses and was most similar to myohaloviruses phiCh1 (host: Natrialba magadii) and phiH1 (host: Hbt. salinarum). It carries a tail-fiber gene module similar to the invertible modules present in phiH1 and phiCh1. However, while the tail genes of ChaoS9 were similar to those of phiCh1 and phiH1, the Mcp of ChaoS9 was most similar (36\% aa identity) to that of Haloarcula hispanica tailed virus 1 (HHTV-1). Provirus elements related to ChaoS9 showed most similarity to tail/assembly proteins but varied in their similarity with head/assembly proteins. The right arm (29-55 kbp) of ChaoS9 encoded proteins involved in DNA replication (ParA, RepH, and Orc1) but the other proteins showed little similarity to those from phiH1, phiCh1, or provirus elements, and most of them could not be assigned a function. ChaoS9 is probably best classified within the genus Myohalovirus, as it shares many characteristics with phiH1 (and phiCh1), including many similar proteins. However, the head/assembly gene region appears to have undergone a recombination event, and the inferred proteins are different to those of phiH1 and phiCh1, including the major capsid protein. This makes the taxonomic classification of ChaoS9 more ambiguous. We also report a revised genome sequence and annotation of Natrialba virus phiCh1.
\end{abstract}

Keywords: halovirus; caudovirus; halobacteria; Archaea; haloarchaea; genome inversion; transposon

\section{Introduction}

Viruses infecting extremely halophilic archaea (haloarchaea) include a variety of morphotypes, such as caudoviruses (e.g., phiH1), round viruses (e.g., SH1), pleomorphic viruses (e.g., His2), and spindle-shaped viruses (e.g., His1) [1,2]. In hypersaline environments such as salt lakes and saltern crystallizer ponds, the vast majority of prokaryotes are usually haloarchaea (Class Halobacteria) with cell concentrations reaching up to $10^{8}$ per $\mathrm{mL}$, but the concentration of virus particles is often 10 -fold 
higher [3], so that haloviruses are important regulators of host cell populations as well as major drivers of their evolution. Among the haloarchaeal caudoviruses, examples of myovirus-like and siphovirus-like viruses have been described, and both temperate and virulent (lytic) isolates have been reported [4-7]. With many haloarchaeal genome sequences now available, it is clear that proviruses are common and widespread, and can be present either as plasmids [8] or integrated into the host chromosome $[9,10]$. The high numbers of viruses in environmental samples, their stability, and the need for large quantities of salt for cultivation of haloarchaea, is a potential hazard for large scale culture of haloarchaea for biotechnological purposes, as virus contamination from medium components or the local environment could result in lysis of the cells.

For many decades, the biological function of bacteriorhodopsin was a focus of study in the Oesterhelt department of the Max Planck Institute (MPI) in Martinsried [11-13]. The bacteriorhodopsin producer strain Halobacterium salinarum strain S9 was derived from strain R1 (DSM 671). The R1 strain and derivative strains such as S9 have been widely used to produce commercial quantities of bacteriorhodopsin [14]. Hbt. salinarum S9 (originally strain R1S9) was first described in a 1979 review by Stoeckenius et al. [15] as a purple membrane overproducer strain derived by Lily Jan (unpublished) from strain R1 by nitrosoguanidine mutagenesis. It has been used in numerous studies, such as gene regulation and expression [16-19], and cell chemotaxis and phototaxis [20,21]. In some studies, this strain has also been labelled as bat+ [18].

Commercial production of pure bacteriorhodopsin entails regular, large-scale cultivation, a practice known to increase the likelihood of virus contamination that can lead to sudden lysis of the microbial cells in a bioreactor [22]. Such an event occurred in 2007 in a $1 \mathrm{~m}^{3}$ culture of Hbt. salinarum S9 being grown at the MPI laboratory. A similar, spontaneous lysis event had occurred in 1974 in the Oesterhelt group and a sample of culture fluid, which had been collected by Hartmut Michel, turned out to contain halovirus phiH [23]. Accordingly, it was initially assumed that phiH was also responsible for the 2007 event.

The aim of this study was to characterize the virus that caused the large-scale lysis of Hbt. salinarum S9 in 2007. This turned out to be a novel halovirus, related to phiH1 and phiCh1, which we named

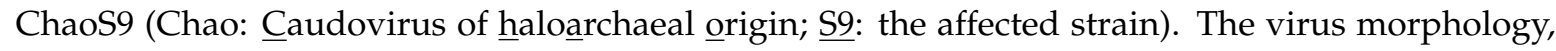
proteins, and genome sequence were analyzed and compared with other described haloviruses and provirus elements in order to assess its novelty, evolution, and taxonomic position. During this study, we also resequenced Natrialba virus phiCh1.

\section{Materials and Methods}

\subsection{Host Strain, Virus Isolation, Cultivation, and Purification}

Hbt. salinarum S9 is a purple-membrane (Pum) constitutive strain [15]. It was grown aerobically in peptone/salts medium, as previously described [24]. Virus purification and DNA extraction followed the methods previously described for halovirus phiH [23]. Briefly, this involved filtration of the lysate through diatomaceous earth (DE), concentration of viral particles from the filtrate by $\mathrm{PEG}_{6000}$ precipitation, and finally, banding twice on $\mathrm{CsCl}$ gradients. The plaque assay method followed that was described previously for phiH [23].

\subsection{DNA Sequencing and Assembly of the ChaoS9 Genome}

The ChaoS9 genome was sequenced by the whole-genome shotgun approach (7-fold coverage). Briefly, DNA was randomly sheared by sonication and fragments cloned into plasmid vectors and sequenced by the chain termination method using the BigDye system (Applied Biosystems, ABI, Foster City, CA, USA). Contig assembly used the Phred-Phrap-Consed package [25]. The remaining gaps were sequenced by targeted PCR amplification of viral DNA using custom primers (Supplementary Table S1), followed by sequencing of the amplimers using the BigDye system. Sequencing was performed at the MPI of Biochemistry core sequencing facility (Martinsried, Germany). 


\subsection{DNA Resequencing of Natrialba Virus phiCh1}

The genome sequence of phiCh1 was determined using the Illumina HiSeq platform (Max-Planck Genome Centre, Cologne, Germany), as previously described for phiH1 [26]. This generated $309 \mathrm{Mbp}$ of high-quality sequence data. Reads were mapped to the reference genome (GenBank:AF440695) using the "map to reference" option within the Geneious (version 10.2) environment, as described for phiH1 [26]. Average genome coverage was 3192-fold. Gene annotation used a combination of gene prediction with GeneMarkS-2 [27] and manual refinement using database searches (BLASTp/BLASTn). Refinement of the annotation took into consideration the original annotation of phiCh1, the annotation of Nab. magadii plasmid pNMAG03 [28] and the annotation of haloviruses phiH1 and ChaoS9, as well as the nr database at the NCBI webserver (https:/ /blast.ncbi.nlm.nih.gov, accessed 10 December 2018).

\subsection{Electron Microscopy of Virus}

Samples of purified ChaoS9 virus were fixed iso-osmotically with $2.5 \%(v / v)$ glutaraldehyde. A drop of the sample was then placed on a carbon-coated copper grid, freshly treated by glow discharge to make it hydrophilic. After incubation for $2 \mathrm{~min}$, the drop was quickly removed, and the grid was stained with a solution of $1 \%(w / v)$ uranyl acetate and $0.01 \%(w / v)$ glucose. Micrographs were taken with an EM 912 electron microscope (Zeiss, Oberkochen, Germany) equipped with an integrated OMEGA energy filter operated in the zero-loss mode. Head diameters were measured on micrographs from vertex-to-vertex, not including the axis in line with the tail.

\subsection{Protein Analyses of Purified Virus}

Samples of purified virus were added to Laemmli sample buffer (with 2-mercaptoethanol) [29] and heated at $95^{\circ} \mathrm{C}$ for $5 \mathrm{~min}$ before loading on a precast NuPAGE $4 \%-12 \%$ Bis-Tris polyacrylamide gel (Invitrogen). The running buffer was NuPAGE MES buffer with $0.1 \%(w / v)$ sodium dodecyl sulfate (SDS). PageRulerTM prestained protein ladder-3 (Fermentas, \#SM1819), containing proteins of 250, $130,100,70,55,35,25,15,10 \mathrm{kDa}$, were loaded in parallel. The gel was stained with Coomassie blue and destained in $10 \%$ acetic acid.

\subsection{Bioinformatics Analyses}

Sequence alignments, editing, and phylogenetic tree reconstructions were performed within the Geneious (version 10.2) suite of programs (https:/ / www.geneious.com/) [30]. For phylogenetic tree reconstructions, protein sequences were first aligned using CLUSTALW, and trees inferred using the Neighbor-Joining algorithm (within Geneious). Consensus trees were determined after 100 bootstrap repetitions. GeneMarkS-2 [27] was used for gene prediction. Protein and DNA sequence similarity searches used the programs BLASTp and BLASTn to search the nr databases at the NCBI webserver (https: / / blast.ncbi.nlm.nih.gov, accessed on 10 December 2018). The VIRFAM webserver (http:/ /biodev.cea.fr/virfam/) [31] was used to classify ChaoS9. Halovirus genomes were compared by the dot plot method zPicture [32]. Average Nucleotide Identity (ANIb) values between viral genomes were calculated using EZbiocloud webserver [33], and a heatmap produced from these values using heatmapper [34]. Searches for matching CRISPR spacers were performed at the CRISPRs web server (http://crispr.i2bc.paris-saclay.fr/crispr/BLAST/CRISPRsBlast.php) [35] and at the IMG/VR server (https://img.jgi.doe.gov/cgi-bin/vr/main.cgi) [36], and also by direct searching of hypersaline metagenomes using the crass software [37], as previously described [26]. Identification of the pac site utilized the program PhageTerm [38] as implemented on the CPT Phage Galaxy (https:/ / cpt.tamu. edu/galaxy-pub/). Correction of the molecular weight estimates of acidic proteins was based on the study of Guan et al. [39]. The following equations were used to convert the protein MW calculated from the inferred protein sequence into an apparent protein MW expected upon SDS-PAGE: $M_{\text {real }}$ $+\mathrm{Sh}=\mathrm{MW}_{\mathrm{app}}$, where $\mathrm{MW}_{\text {real }}$ is the MW computed from the protein sequence, $\mathrm{MW}_{\mathrm{app}}$ is the MW expected to be observed by SDS-PAGE, and Sh is the shift (or estimated error) computed by the formula 
Sh $=$ len $\times(276.5 x-31.33)$. Here, len is the length of the protein (in aa) and $x$ represents the proportion of acidic amino acids (Glu, Asp).

\section{Data Availability}

The ChaoS9 genome sequence has been deposited at Genbank under the accession MK310226. The revised phiCh1 genome sequence has been deposited under accession MK450543 and the phiCh1 raw reads were submitted to the SRA and can be retrieved via BioProject PRJNA517034.

\section{Results}

\subsection{Isolation of Halovirus ChaoS9}

In 2007, a large-scale culture $\left(1 \mathrm{~m}^{3}\right)$ of Hbt. salinarum S9 lysed spontaneously. Suspecting a virus infection, the lysate was processed by the method described for purifying halovirus phiH1 [23]. This involved filtration through diatomaceous earth, addition of $\mathrm{PEG}_{6000}$ to precipitate virus particles, and the resulting pellets applied to $\mathrm{CsCl}$ gradients. Virus bands were observed on $\mathrm{CsCl}$ gradients, and negative-stain electron-microscopy of this fraction revealed a homogeneous population of tailed virus particles, some displaying contracted tails (Figure 1). The head diameter was $61 \mathrm{~nm}$, uncontracted tails were $128 \times 17 \mathrm{~nm}$, and contracted tails had sheaths of $74 \times 23 \mathrm{~nm}$.

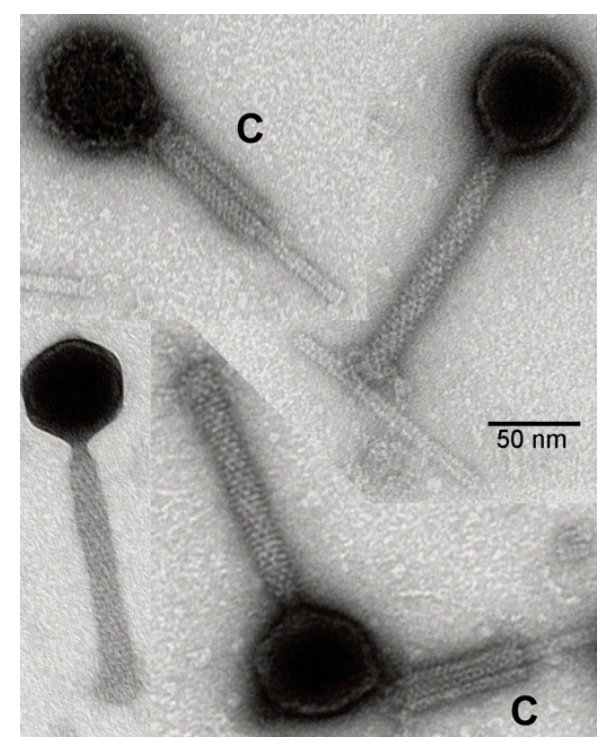

Figure 1. Morphology of ChaoS9 particles by negative-stain electron microscopy. Purified virus was fixed with $2.5 \%$ glutaraldehyde, negatively-stained with $1 \%$ uranyl acetate and examined under a Zeiss EM 912 electron microscope. Scale bar represents $50 \mathrm{~nm}$. Examples of contracted tails (labeled with a C) are shown in the top left-hand corner and the bottom right-hand corner.

\subsection{Virus Proteins}

The proteins of purified virus were separated by SDS-PAGE and revealed four major protein bands (VP1, VP3, VP4, and VP7), three minor bands (VP2, VP5, and VP6), and several very faint bands (Figure 2). 


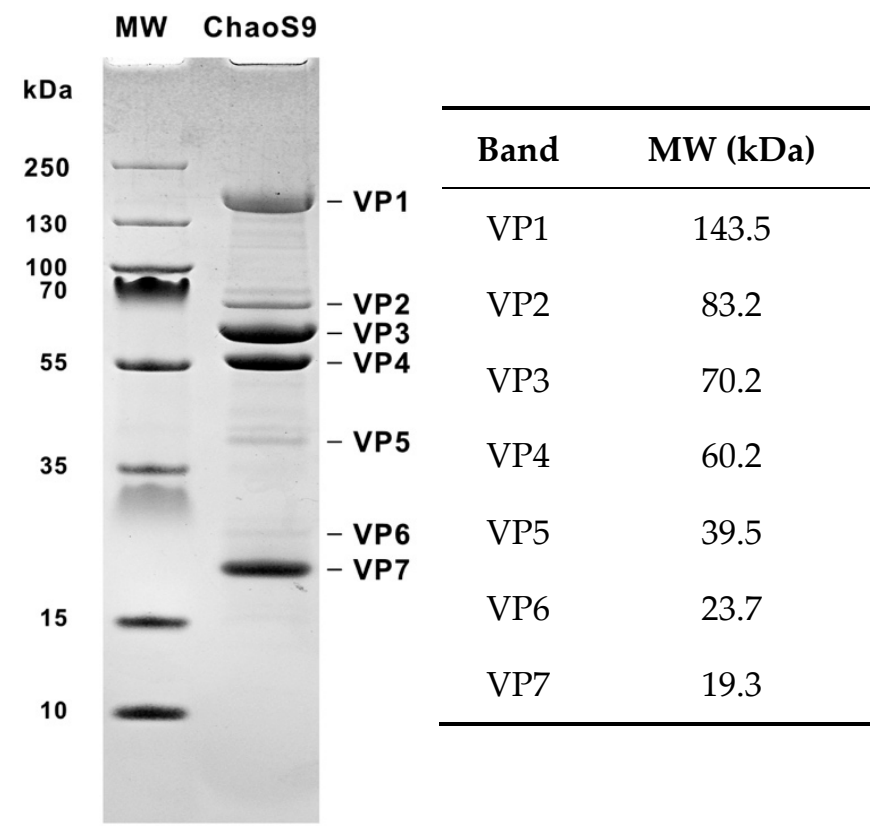

Figure 2. Proteins of halovirus ChaoS9. Purified virus was heated in sample buffer and separated on a $4 \%-12 \%$ SDS-polyacrylamide gel, and the separated proteins stained with Coomassie blue. MW, molecular weight standards (PageRuler). The sizes of the protein standards are indicated at the left (in $\mathrm{kDa}$ ). Virus proteins (VP1 to VP7) are numbered from largest to smallest. Molecular weight estimates of the major virus proteins are shown in the adjacent table.

\subsection{ChaoS9 Genome and Sequence}

Nucleic acids were extracted from virus preparations, treated with several restriction enzymes and the digests separated by agarose gel electrophoresis (Figure 3). Cleavage of the viral genome by these enzymes, and the different fragment patterns observed for each enzyme, indicated that the genome was dsDNA. The ChaoS9 genome was sequenced using the whole-genome shotgun approach (7-fold coverage; see Methods) and contig gaps were closed by PCR amplification of viral DNA using specific primers (Table S1). All sequences assembled to a single contig with a unit length of 55,145 nt and a $\mathrm{G}+\mathrm{C}$ content of $65.3 \%$ (Table 1). Comparison of the observed restriction fragment patterns with in silico predictions based on linear and circular versions of the genome (Figure S1) not only showed a close correspondence, but also identified terminal fragments that were either underrepresented or not visible (white triangles) on gels, as well as bands predicted to occur only in longer than unit length genomes (blue triangles). These results were consistent with the viral genome being partially redundant and circularly permuted, as is typical for headful packaging. 


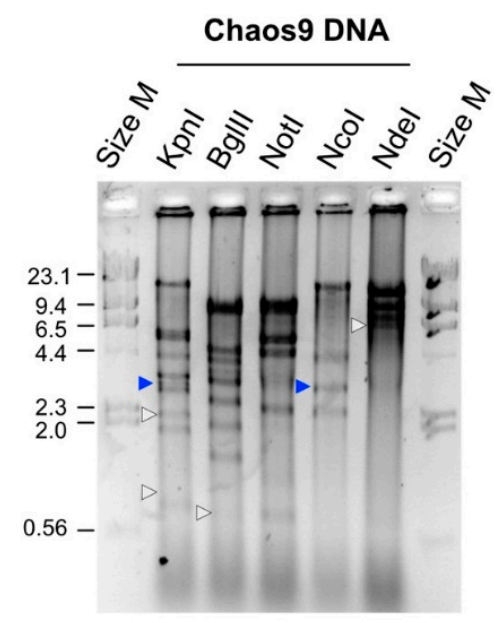

Figure 3. Restriction digests of ChaoS9 DNA. Enzymes used are indicated above each well. The outside wells (Size M) were loaded with DNA size markers (Lambda-HindIII), and the fragment sizes (in kbp) are shown at the left edge. White triangles; terminal fragments predicted from the DNA sequence that were either underrepresented or not visible on gels. Blue triangles; bands predicted from the DNA sequence to occur only in longer than unit length genomes. See also Supplementary Figure S1.

A dotplot comparison of this sequence with 17 other tailed haloviruses showed a specific and close relationship with phiCh1 and phiH1 (Figure 4a), and average nucleotide identity ( $\mathrm{ANIb}$ ) values between ChaoS9, phiCh1 and phiH1 were $\geq 74 \%$ (Figure $4 \mathrm{~b}$ ). In a recent study, the complete genome sequence of phiH1 was compared to the previously published sequence of phiCh1 [26], and they shared $63 \%$ (BLASTn) nucleotide identity. As part of the present study, we have resequenced phiCh1 (see below and Table S2 for details). Since phiH1 is a valid species of the genus Myohalovirus [40], phiCh1 should be placed in the same genus. While phiCh1 and phiH1 show strong similarity over most of their genomes, their similarity to ChaoS9 is largely confined to a central region covering from about $10-30 \mathrm{kbp}$. This can be seen in Figure $4 \mathrm{a}$, but is more clearly evident in the annotated genome comparison depicted in Figure 5.

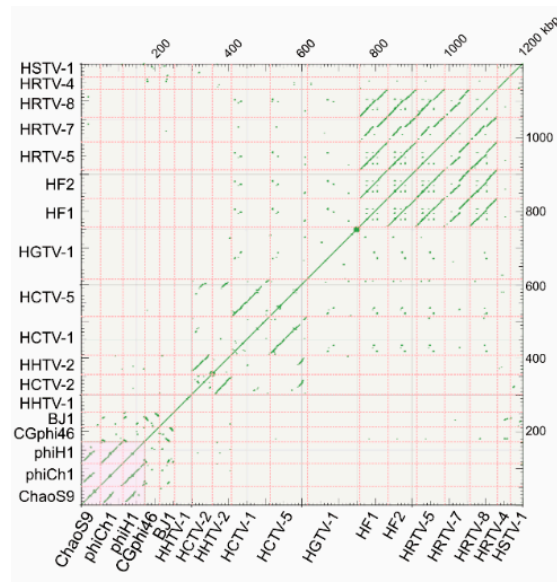

(a)

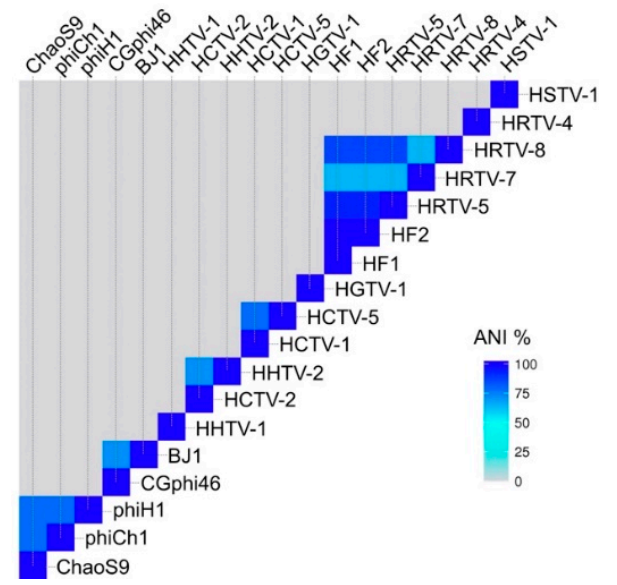

(b)

Figure 4. Comparison of the ChaoS9 genome with the genomes of 17 other published halovirus isolates. (a) Dot plot showing sequence similarity using the method of zPicture (Blastz) [32]. Names of viruses are shown along lower and left edges, and their lengths are indicated by the pink dashed lines. The scale, in $\mathrm{kbp}$, is shown along the upper and right edges. The pink shaded box at lower left highlights the similarity between ChaoS9, phiCh1 and phiH1. (b) Average Nucleotide Identity (ANIb) heatmap. ANIb percentage values were calculated using EZbiocloud [33] and the heatmap produced using heatmapper [34]. Color scale indicates \% ANIb. 


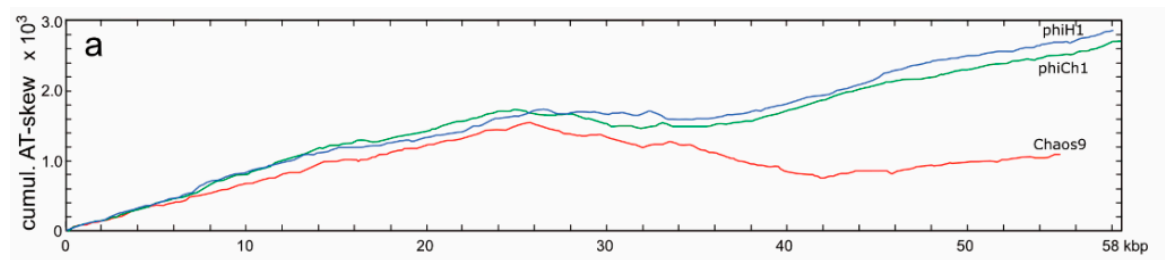

b phiCh1 os ov $e^{6}$

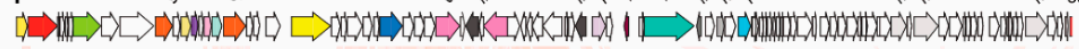

c Chaos9

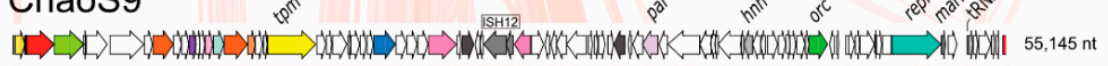
so

d phiH1

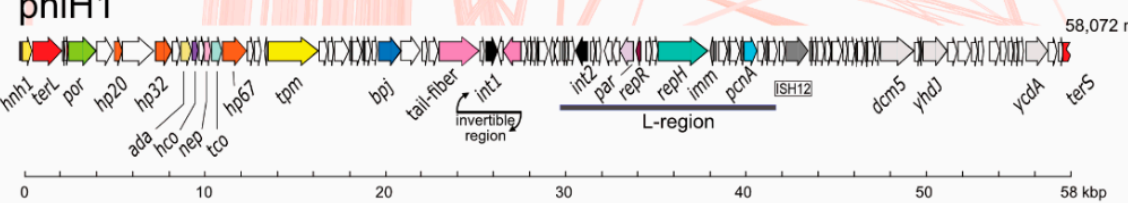

Figure 5. Genome map of ChaoS9 compared to phiCh1 and phiH1. (a) Cumulative AT-skew of all three viral genomes. (b) Genome map of phiCh1; (c) Genome map of ChaoS9; (d) Genome map of phiH1. Scale bar at bottom shows length, in kbp. Pink shading between genome maps indicates regions of similarity (tBLASTx, $\geq 30 \%$ amino acid identity). Functionally similar genes have been colored the same and are labeled nearby on one or more of the genomes (see Table 2 for details). The colors, gene labels (and encoded proteins) are: red, terL (large subunit terminase); light green, por (portal protein); brown, capsid protein genes, such as $m c p, g p E$ and $h p 32$ (major capsid protein), hp20, hp67, cp67; yellow, tpm (tape measure protein); blue, bpj (baseplate J family protein); pink, tail fiber genes; light purple, par (plasmid partition); dark green, orc1, (replication protein Orc1); light blue, repH (plasmid replication protein); light grey, $d c m 5, y h d J, y c d A, m . I I, m . I I I, m . I$ (methyltransferases); dark grey, ISH12 transposase; black, integrases (int1, int2). Genes of unknown or uncertain function are uncolored (white). The L-region of phiH1 has been described by [41].

Table 1. Characteristics of ChaoS9 and related haloviruses phiH1 and phiCh1.

\begin{tabular}{|c|c|c|c|c|c|c|c|c|}
\hline Virus $^{1}$ & $\begin{array}{c}\text { Head } \\
\text { Diameter } \\
(\mathrm{nm})\end{array}$ & $\begin{array}{l}\text { Tail Length } \times \\
\text { Width }(\mathrm{nm})\end{array}$ & Morphotype & $\begin{array}{c}\text { Plaque } \\
\text { Morphology }\end{array}$ & $\begin{array}{l}\text { Unit Genome } \\
\text { Length }^{2} \text { (nt) }\end{array}$ & $\% \mathrm{G}+\mathrm{C}$ & $\begin{array}{l}\text { GenomeEnds } \\
\text { in Virus }^{3}\end{array}$ & $\begin{array}{l}\text { Temperate } \\
\text { (Genome } \\
\text { Form) }\end{array}$ \\
\hline ChaoS9 & 61 & $128 \times 17$ & myovirus & turbid & 55,145 & 65.3 & $\begin{array}{c}\text { ds, linear, TR, } \\
\mathrm{CP},>1 \text { unit } \\
\text { length }\end{array}$ & $?$ \\
\hline phiH1 & 64 & $170 \times 18$ & myovirus & turbid & 58,072 & 63.7 & $\begin{array}{c}\text { ds, linear, TR, } \\
\mathrm{CP},>1 \text { unit } \\
\text { length }\end{array}$ & $\begin{array}{l}\text { Yes, provirus } \\
\text { is a plasmid } \\
\text { (circular, ds, } 1 \\
\text { unit length) }\end{array}$ \\
\hline phiCh1 & 70 & $130 \times 20$ & myovirus & turbid & 58,487 & 61.9 & $\begin{array}{c}\text { ds, linear, TR, } \\
\mathrm{CP},>1 \text { unit } \\
\text { length }\end{array}$ & $\begin{array}{l}\text { Yes, provirus } \\
\text { is a plasmid } \\
\text { (circular, ds, } 1 \\
\text { unit length) }\end{array}$ \\
\hline
\end{tabular}

${ }^{1}$ Data from this study for ChaoS9, [23] for phiH and [42] for phiCh1. ${ }^{2}$ Data from this study (ChaoS9), Dyall-Smith et al. [26] (phiH1), and Witte et al., and this study [43] (phiCh1). ${ }^{3} \mathrm{TR}, \mathrm{CP}$; terminally redundant, circularly permuted. 
Table 2. Annotated coding sequences (CDS) of the ChaoS9 genome (accession MK450543).

\begin{tabular}{|c|c|c|c|c|c|c|c|c|}
\hline Start (nt) & Stop (nt) & Locus_Tag & Length (bp) & Direction & Gene & Product & Homologs $^{1}$ : phiCh1, pNMAG03 & $\begin{array}{c}\text { Homologs }^{2} \text { : phiH1, } \\
\text { [Other] }\end{array}$ \\
\hline 100 & 669 & ChaoS9_005 & 570 & + & - & HTH domain protein & $\begin{array}{l}\text { PhiCh1_005, PhiCh1p02, ORF1, } \\
\text { Nmag_4251 }\end{array}$ & PhiH1_005 \\
\hline 656 & 2302 & ChaoS9_010 & 1647 & + & terL & terminase large subunit TerL & - & [HALG_00007] \\
\hline 2316 & 3944 & ChaoS9_015 & 1629 & + & por & portal protein Por & - & [HGTV1_7] \\
\hline 3937 & 4083 & ChaoS9_020 & 147 & + & - & CxxC motif protein & - & ORPHAN \\
\hline 4086 & 5273 & ChaoS9_025 & 1188 & + & - & $\begin{array}{l}\text { putative phage head assembly } \\
\text { protein, SPP1_gp7 family }\end{array}$ & - & [C478_10461] \\
\hline 5384 & 7300 & ChaoS9_030 & 1917 & + & - & probable prohead protease protein & - & [HLASA_2034] \\
\hline 7303 & 7755 & ChaoS9_035 & 453 & + & - & uncharacterized protein & - & [HLASA_2033] \\
\hline 7801 & 8928 & ChaoS9_040 & 1128 & + & - & major capsid protein $\mathrm{MCP}$ & - & $\begin{array}{l}{ }^{3} \text { [HLASA_2032; } \\
\text { HHTV1_21] }\end{array}$ \\
\hline 8944 & 9363 & ChaoS9_045 & 420 & + & - & uncharacterized protein & $\begin{array}{l}\text { PhiCh1_055, PhiCh1p13, ORF12, } \\
\text { Nmag_4261 }\end{array}$ & $\begin{array}{l}\text { 6 PhiH1_050, } \\
\text { [HLASA_2031] }\end{array}$ \\
\hline 9398 & 9784 & ChaoS9_050 & 387 & + & - & uncharacterized protein & - & [HLASA_2030] \\
\hline 9781 & 10191 & ChaoS9_055 & 411 & + & hco & head closure protein Hco, type 1 & $\begin{array}{c}\text { PhiCh1_065, PhiCh1p15, ORF14, } \\
\text { Nmag_4263 }\end{array}$ & $\begin{array}{l}{ }^{5} \text { PhiH1_060, } \\
\text { [WP_054519912] }\end{array}$ \\
\hline 10188 & 10421 & ChaoS9_060 & 234 & + & - & uncharacterized protein & - & ORPHAN \\
\hline 10414 & 10701 & ChaoS9_065 & 288 & + & - & uncharacterized protein & $\begin{array}{l}\text { PhiCh1_070, PhiCh1p16, ORF15, } \\
\text { Nmag_4264 }\end{array}$ & $\begin{array}{l}\text { 6 PhiH1_065, } \\
\text { [HLASA_2029] }\end{array}$ \\
\hline 10703 & 11149 & ChaoS9_070 & 447 & + & nep & putative neck protein Nep, type 1 & $\begin{array}{l}\text { PhiCh1_075, PhiCh1p17, ORF16, } \\
\text { Nmag_4265 }\end{array}$ & $\begin{array}{c}\text { PhiH1_070 } \\
\text { [HLASA_2028] }\end{array}$ \\
\hline 11156 & 11746 & ChaoS9_075 & 591 & + & tco & tail completion protein Tco, type 1 & $\begin{array}{l}\text { PhiCh1_080, PhiCh1p18, ORF17, } \\
\text { Nmag_4266 }\end{array}$ & $\begin{array}{c}\text { PhiH1_075 } \\
\text { [HLASA_2027] } \\
\end{array}$ \\
\hline 11767 & 13071 & ChaoS9_080 & 1305 & + & - & tail sheath protein & $\begin{array}{c}\text { PhiCh1_085, PhiCh1p19, ORF18, } \\
\text { Nmag_4267 }\end{array}$ & $\begin{array}{l}\text { PhiH1_080 } \\
\text { [HLASA_2026] }\end{array}$ \\
\hline
\end{tabular}


Table 2. Cont.

\begin{tabular}{|c|c|c|c|c|c|c|c|c|}
\hline Start (nt) & Stop (nt) & Locus_Tag & Length (bp) & Direction & Gene & Product & Homologs $^{1}$ : phiCh1, pNMAG03 & $\begin{array}{c}\text { Homologs }{ }^{2}: \text { phiH1, } \\
\text { [Other] }\end{array}$ \\
\hline 13082 & 13480 & ChaoS9_085 & 399 & + & - & predicted tail tube protein & $\begin{array}{l}\text { PhiCh1_090, PhiCh1p20, ORF19, } \\
\text { Nmag_4268 }\end{array}$ & $\begin{array}{c}\text { PhiH1_085 } \\
\text { [WP_054519907] }\end{array}$ \\
\hline 13492 & 13932 & ChaoS9_090 & 441 & + & - & uncharacterized protein & $\begin{array}{c}\text { PhiCh1_095, PhiCh1p21, ORF20, } \\
\text { Nmag_4269 }\end{array}$ & $\begin{array}{c}\text { PhiH1_090 } \\
\text { [HLASA_2025] } \\
\end{array}$ \\
\hline 13935 & 14144 & ChaoS9_095 & 210 & + & - & uncharacterized protein & - & [WP_054519905] \\
\hline 14147 & 16891 & ChaoS9_100 & 2745 & + & tpm & tape-measure tail protein Tpm & $\begin{array}{c}7 \text { PhiCh1_105, } \\
\text { PhiCh1p23+PhiCh1p24, } \\
\text { ORF22+ORF23, Nmag_4272 }\end{array}$ & $\begin{array}{l}\text { PhiH1_100 } \\
\text { [HLASA_2024] }\end{array}$ \\
\hline 16895 & 17422 & ChaoS9_105 & 528 & + & - & uncharacterized protein & $\begin{array}{l}\text { PhiCh1_110, PhiCh1p25, ORF24, } \\
\text { Nmag_4273 }\end{array}$ & $\begin{array}{c}\text { PhiH1_105 } \\
\text { [HLASA_2023] }\end{array}$ \\
\hline 17423 & 17767 & ChaoS9_110 & 345 & + & - & uncharacterized protein & $\begin{array}{l}\text { PhiCh1_115, PhiCh1p26, ORF25, } \\
\text { Nmag_4274 }\end{array}$ & $\begin{array}{c}\text { PhiH1_110 } \\
\text { [HLASA_2022] }\end{array}$ \\
\hline 17771 & 18604 & ChaoS9_115 & 834 & + & - & uncharacterized protein & $\begin{array}{c}7 \text { PhiCh1_120, } \\
\text { PhiCh1p27+PhiCh1p28, } \\
\text { ORF26+ORF27, Nmag_4275 }\end{array}$ & $\begin{array}{l}\text { PhiH1_115 } \\
\text { [HLASA_2021] }\end{array}$ \\
\hline 18644 & 18787 & ChaoS9_120 & 144 & + & - & CxxC motif protein & - & PhiH1_120 \\
\hline 18784 & 19335 & ChaoS9_125 & 552 & + & - & uncharacterized protein & $\begin{array}{l}\text { PhiCh1_125, PhiCh1p29, ORF28, } \\
\text { Nmag_4276 }\end{array}$ & $\begin{array}{c}\text { PhiH1_125 } \\
\text { [HLASA_2020] }\end{array}$ \\
\hline 19338 & 19700 & ChaoS9_130 & 363 & + & - & virus-related protein & - & PhiH1_135 \\
\hline 19697 & 20062 & ChaoS9_135 & 366 & + & - & uncharacterized protein & $\begin{array}{l}\text { PhiCh1_130, PhiCh1p30, ORF29, } \\
\text { Nmag_4277 }\end{array}$ & $\begin{array}{c}\text { PhiH1_140 } \\
\text { [HLASA_2019] }\end{array}$ \\
\hline 20069 & 21328 & ChaoS9_140 & 1260 & + & $b p j$ & baseplate J family protein Bpj & $\begin{array}{l}\text { PhiCh1_135, PhiCh1p31, ORF30, } \\
\text { Nmag_4278 }\end{array}$ & $\begin{array}{c}\text { PhiH1_145 } \\
\text { [HLASA_2018] } \\
\end{array}$ \\
\hline 21321 & 21929 & ChaoS9_145 & 609 & + & - & uncharacterized protein & $\begin{array}{c}\text { PhiCh1_140, PhiCh1p32, ORF31, } \\
\text { Nmag_4279 }\end{array}$ & $\begin{array}{c}\text { PhiH1_150 } \\
\text { [HLASA_2017] }\end{array}$ \\
\hline
\end{tabular}


Table 2. Cont

\begin{tabular}{|c|c|c|c|c|c|c|c|c|}
\hline Start (nt) & Stop (nt) & Locus_Tag & Length (bp) & Direction & Gene & Product & Homologs ${ }^{1}$ : phiCh1, pNMAG03 & $\begin{array}{c}\text { Homologs }{ }^{2}: \text { phiH1, } \\
\text { [Other] }\end{array}$ \\
\hline 21933 & 22517 & ChaoS9_150 & 585 & + & - & uncharacterized protein & $\begin{array}{l}\text { PhiCh1_145, PhiCh1p33, ORF32, } \\
\text { Nmag_4280 }\end{array}$ & [HLASA_2016] \\
\hline 22514 & 23122 & ChaoS9_155 & 609 & + & - & uncharacterized protein & $\begin{array}{c}\text { PhiCh1_150, PhiCh1p34, ORF33, } \\
\text { Nmag_4281 }\end{array}$ & [HLASA_2015] \\
\hline 23115 & 24698 & ChaoS9_160 & 1584 & + & - & repeat-containing tail fiber protein & $\begin{array}{c}8 \text { PhiCh1_155+ PhiCh1_175, } \\
\text { PhiCh1p35+PhiCh1p37, } \\
\text { ORF34+ORF36, } \\
\text { Nmag_4282+Nmag_4286 }\end{array}$ & $\begin{array}{l}\text { PhiH1_165+ } \\
\text { PhiH1_185 }\end{array}$ \\
\hline 24702 & 24986 & ChaoS9_165 & 285 & + & - & uncharacterized protein & $\begin{array}{c}8 \text { PhiCh1_160+ PhiCh1_170, } \\
\text { Nmag_4285+Nmag_4283 }\end{array}$ & $\begin{array}{l}\text { PhiH1_180+ } \\
\text { PhiH1_170 }\end{array}$ \\
\hline 25024 & 25698 & ChaoS9_170 & 675 & + & int1 & $\begin{array}{l}\text { tyrosine integrase/recombinase } \\
\text { Int1 }\end{array}$ & $\begin{array}{l}\text { PhiCh1_165, PhiCh1p36, ORF35, } \\
\text { Nmag_4284 }\end{array}$ & PhiH1_175 \\
\hline 25709 & 25996 & ChaoS9_175 & 288 & - & - & uncharacterized protein & $\begin{array}{l}8 \text { PhiCh1_160+ PhiCh1_170, } \\
\text { Nmag_4285+Nmag_4283 }\end{array}$ & $\begin{array}{l}\text { PhiH1_180+ } \\
\text { PhiH1_170 }\end{array}$ \\
\hline 25999 & 26145 & ChaoS9_180 & 147 & - & - & $\begin{array}{l}\text { repeat-containing tail fiber protein } \\
\text { (C-term) (nonfunctional) }^{9}\end{array}$ & $* 9$ & $* 9$ \\
\hline 26199 & 27455 & ChaoS9_185 & 1257 & - & $\operatorname{tnp} B$ & IS1341-type transposase TnpB & - & PhiH1_340 \\
\hline 27457 & 27849 & ChaoS9_190 & 393 & - & $\operatorname{tnp} A$ & IS200-type transposase TnpA & - & PhiH1_335 \\
\hline 27906 & 28820 & ChaoS9_195 & 915 & - & - & $\begin{array}{l}\text { repeat-containing tail fiber protein } \\
\text { (N-term) (nonfunctional) }\end{array}$ & $\begin{array}{c}\text { 8 PhiCh1_155+PhiCh1_175, } \\
\text { PhiCh1p35+PhiCh1p37, } \\
\text { ORF34+ORF36, } \\
\text { Nmag_4282+Nmag_4286 }\end{array}$ & $\begin{array}{l}\text { PhiH1_165+ } \\
\text { PhiH1_185 }\end{array}$ \\
\hline 28854 & 29579 & ChaoS9_200 & 726 & + & - & transmembrane domain protein & $\begin{array}{l}\text { PhiCh1_180, PhiCh1p38, ORF37, } \\
\text { Nmag_4287 }\end{array}$ & - \\
\hline 29589 & 29861 & ChaoS9_205 & 273 & - & - & HTH domain protein & $\begin{array}{c}\text { PhiCh1_185, PhiCh1p39, ORF38, } \\
\text { Nmag_4288 }\end{array}$ & - \\
\hline
\end{tabular}


Table 2. Cont

\begin{tabular}{|c|c|c|c|c|c|c|c|c|}
\hline Start (nt) & Stop (nt) & Locus_Tag & Length $(b p)$ & Direction & Gene & Product & Homologs ${ }^{1}$ : phiCh1, pNMAG03 & $\begin{array}{c}\text { Homologs }{ }^{2} \text { : phiH1, } \\
\text { [Other] }\end{array}$ \\
\hline 29933 & 30241 & ChaoS9_210 & 309 & - & - & uncharacterized protein & $\begin{array}{c}\text { PhiCh1_190, PhiCh1p40, ORF39, } \\
\text { Nmag_4289 }\end{array}$ & PhiH1_220 \\
\hline 30238 & 30813 & ChaoS9_215 & 576 & - & - & $\begin{array}{l}\text { glutamine amidotransferase } \\
\text { domain protein, class-II }\end{array}$ & $\begin{array}{c}\text { PhiCh1_195, PhiCh1p41, ORF40, } \\
\text { Nmag_4290 }\end{array}$ & - \\
\hline 30818 & 31891 & ChaoS9_220 & 1074 & - & - & uncharacterized protein & $\begin{array}{c}\text { 7 PhiCh1_200, } \\
\text { PhiCh1p42+PhiCh1p43, } \\
\text { ORF41+ORF42, Nmag_4291 }\end{array}$ & - \\
\hline 32030 & 32266 & ChaoS9_225 & 237 & + & - & uncharacterized protein & - & ORPHAN \\
\hline 32339 & 32584 & ChaoS9_230 & 246 & + & - & uncharacterized protein & - & PhiH1_225 \\
\hline 32581 & 33009 & ChaoS9_235 & 429 & + & - & VapC family toxin & - & [BRC75_08225] \\
\hline 33098 & 33448 & ChaoS9_240 & 351 & + & - & uncharacterized protein & PhiCh1_230, Nmag_4297 & PhiH1_250 \\
\hline 33457 & 34059 & ChaoS9_245 & 603 & - & int2 & $\begin{array}{l}\text { tyrosine integrase/recombinase } \\
\text { Int2 }\end{array}$ & $\begin{array}{c}\text { PhiCh1_215, PhiCh1p46, ORF45, } \\
\text { Nmag_4294 }\end{array}$ & PhiH1_240 \\
\hline 34241 & 34432 & ChaoS9_250 & 192 & - & - & uncharacterized protein & - & ORPHAN \\
\hline 34507 & 35055 & ChaoS9_255 & 549 & - & - & uncharacterized protein & - & $\begin{array}{c}\text { PhiH1_255 } \\
\text { [C466_00612] }\end{array}$ \\
\hline 35048 & 35902 & ChaoS9_260 & 855 & - & - & Plasmid partition protein ParA & $\begin{array}{c}\text { PhiCh1_220, PhiCh1p47, ORF46, } \\
\text { Nmag_4295 }\end{array}$ & PhiH1_265 \\
\hline 35976 & 36440 & ChaoS9_265 & 465 & - & - & transmembrane domain protein & - & PhiH1_210 \\
\hline 36460 & 38241 & ChaoS9_270 & 1782 & - & - & uncharacterized protein & - & [AV929_12240] \\
\hline 38243 & 38857 & ChaoS9_275 & 615 & - & - & uncharacterized protein & - & [CRI94_04435] \\
\hline 38850 & 39080 & ChaoS9_280 & 231 & - & - & CxxC motif protein & - & [HALLA_11930] \\
\hline 39073 & 39240 & ChaoS9_285 & 168 & - & - & transmembrane domain protein & - & ORPHAN \\
\hline 39233 & 40492 & ChaoS9_290 & 1260 & - & - & uncharacterized protein & - & [DM826_07215] \\
\hline 40485 & 40673 & ChaoS9_295 & 189 & - & - & $\mathrm{CxxC}$ motif protein & - & ORPHAN \\
\hline
\end{tabular}


Table 2. Cont

\begin{tabular}{|c|c|c|c|c|c|c|c|c|}
\hline Start (nt) & Stop (nt) & Locus_Tag & Length $(b p)$ & Direction & Gene & Product & Homologs ${ }^{1}$ : phiCh1, pNMAG03 & $\begin{array}{c}\text { Homologs }{ }^{2}: \text { phiH1, } \\
\text { [Other] }\end{array}$ \\
\hline 40663 & 41181 & ChaoS9_300 & 519 & - & - & uncharacterized protein & - & [DJ71_18565] \\
\hline 41174 & 41611 & ChaoS9_305 & 438 & - & - & $\begin{array}{l}\text { HNH-type endonuclease/MarR } \\
\text { family transcription regulator }\end{array}$ & - & $\begin{array}{l}\text { [DJ70_12900; } \\
\text { B4589_07635] }\end{array}$ \\
\hline 41613 & 41870 & ChaoS9_310 & 258 & - & - & HTH domain protein & - & [Natpe_3999] \\
\hline 41997 & 42602 & ChaoS9_315 & 606 & + & - & uncharacterized protein & - & [C480_10020] \\
\hline 42605 & 43051 & ChaoS9_320 & 447 & + & - & uncharacterized protein & - & [Natgr_3468] \\
\hline 43044 & 43250 & ChaoS9_325 & 207 & + & - & uncharacterized protein & - & ORPHAN \\
\hline 43250 & 43621 & ChaoS9_330 & 372 & + & - & uncharacterized protein & - & [OSG_eHP13_00215] \\
\hline 43621 & 44127 & ChaoS9_335 & 507 & + & - & uncharacterized protein & - & ORPHAN \\
\hline 44124 & 44255 & ChaoS9_340 & 132 & + & - & CxxC motif protein & - & [SAMN04488133_0114] \\
\hline 44342 & 45400 & ChaoS9_345 & 1059 & + & orc1 & Orc1-type DNA replication protein & - & [HLASA_2006] \\
\hline 45482 & 45709 & ChaoS9_350 & 228 & - & - & uncharacterized protein & - & ORPHAN \\
\hline 45837 & 45992 & ChaoS9_355 & 156 & + & - & uncharacterized protein & - & [HALDL1_16575] \\
\hline 46369 & 46905 & ChaoS9_360 & 537 & + & - & uncharacterized protein & $\begin{array}{c}\text { PhiCh1_295, PhiCh1p65, ORF64, } \\
\text { Nmag_4216 }\end{array}$ & 5 [C472_00499] \\
\hline 46902 & 47177 & ChaoS9_365 & 276 & + & - & uncharacterized protein & - & ORPHAN \\
\hline 47179 & 47979 & ChaoS9_370 & 801 & + & - & zinc-finger domain protein & - & [DJ84_18225] \\
\hline 47976 & 48068 & ChaoS9_375 & 93 & + & - & uncharacterized protein & - & ORPHAN \\
\hline 48065 & 48397 & ChaoS9_380 & 333 & + & - & uncharacterized protein & - & ORPHAN \\
\hline 48394 & 51690 & ChaoS9_385 & 3297 & + & repH & plasmid replication protein $\mathrm{RepH}$ & $\begin{array}{c}\text { PhiCh1_245, PhiCh1p55, ORF54, } \\
\text { Nmag_4299 }\end{array}$ & PhiH1_285 \\
\hline 51683 & 51925 & ChaoS9_390 & 243 & + & - & $\begin{array}{l}\text { MarR family transcription } \\
\text { regulator }\end{array}$ & - & [AV929_12115] \\
\hline 51932 & 52591 & ChaoS9_395 & 660 & + & - & CxxC motif protein & - & [C443_17983] \\
\hline 53208 & 53453 & ChaoS9_400 & 246 & + & - & transmembrane domain protein & $\begin{array}{c}\text { PhiCh1_440, PhiCh1p93, ORF92, } \\
\text { Nmag_4244 }\end{array}$ & PhiH1_460 \\
\hline
\end{tabular}


Table 2. Cont.

\begin{tabular}{|c|c|c|c|c|c|c|c|c|}
\hline Start (nt) & Stop (nt) & Locus_Tag & Length (bp) & Direction & Gene & Product & Homologs ${ }^{1}$ : phiCh1, pNMAG03 & $\begin{array}{c}\text { Homologs }^{2} \text { : phiH1, } \\
\text { [Other] }\end{array}$ \\
\hline 53446 & 53769 & ChaoS9_405 & 324 & + & - & transmembrane domain protein & $\begin{array}{c}\text { PhiCh1_445, PhiCh1p94, ORF93, } \\
\text { Nmag_4245 }\end{array}$ & ${ }^{10}$ PhiH1_465 \\
\hline 53766 & 54455 & ChaoS9_410 & 690 & + & - & uncharacterized protein & - & $\begin{array}{c}{ }^{3} \text { [halTADL_2427; } \\
\text { HGTV1_34] }\end{array}$ \\
\hline 54460 & 54705 & ChaoS9_415 & 246 & + & - & DUF217 domain protein & $\begin{array}{l}\text { PhiCh1_460, PhiCh1p97, ORF96, } \\
\text { Nmag_4248 }\end{array}$ & - \\
\hline 54734 & 54922 & ChaoS9_420 & 189 & + & - & $\mathrm{CxxC}$ motif protein & $\begin{array}{c}\text { PhiCh1_465, PhiCh1p98, ORF97, } \\
\text { Nmag_4249 }\end{array}$ & PhiH1_480 \\
\hline $54915>$ & $<63$ & ChaoS9_425 & 294 & + & ters & terminase small subunit TerS & $\begin{array}{c}\text { PhiCh1_470, PhiCh1p01, ORF98, } \\
\text { Nmag_4250 }\end{array}$ & PhiH1_485 \\
\hline
\end{tabular}

${ }^{1}$ PhiCh1/pNMAG03 homologs of ChaoS9 proteins. For phiCh1, three codes are given: the locus tag from the revised genome (PhiCh1_), the RefSeq PhiCh1p and the originally assigned ORF codes (ORF for open reading frame). For example, PhiCh1_005, PhiCh1p02, Orf1. Codes starting with PhiCh1_represent the revised genome sequence and annotation (Genbank accession MK450543, this pution part being encoded at the end of the genome, and the C-terminal part at its beginning. This gene is ORF98 in the original annotation and PhiCh1p01 in the RefSeq annotation. Codes part bing with Nmag represent pNMA else "othe his the phiH1 but exists in phiCh1, this is indicated by a hyphen; if a homolog is lacking in both, phiH1 and phiCh1, then an existing "other" homolog is listed in square brackets; codes are either from UniProt (locus tags) or from NCBI nr (WP numbers). The term ORPHAN indicates a complete lack of homologs. ${ }^{3}$ Multiple homologs, separated by semicolon, are listed when a homolog is found in a halovirus, but this is significantly more distant than the closest homolog. ${ }^{4}$ Multiple homologs indicate a ChaoS9-specific gene fusion. ${ }^{5}$ The combination of phiH1/phiCh1 and "other" homologs is used when the homologs from phiH1 or phiCh1 are especially distant. ${ }^{6}$ The combination of phiH1/phiCh1 and "other" homologs is used for HLASA_codes to illustrate a longer stretch of synteny to PVHS1 from Halanaeroarchaeum sulfurireducens (see later). ${ }^{7}$ Multiple PhiCh1p/ORF codes indicate that the gene was split by a frameshift in the originally published genome sequence of that virus. ${ }^{8}$ Multiple PhiCh1/Nmag codes indicate the existence of paralogs. ${ }^{9}$ This ORF represents the C-terminal fragment of a pseudogene (indicated by the term nonfunctional) which has been targeted by ISH12. The asterisks $\left.{ }^{*}\right)$ indicate that corresponding pseudogene fragments do not exist as independent ORFs in phiH1 or phiCh1. ${ }^{10}$ PhiCh1_445 and PhiH1_465, like ChaoS9 405 , have three predicted transmembrane domains and are suspected to function as a holin [43] 
The pac site of the ChaoS9 genome was identified by alignment with phiH1 and phiCh1, for which pac sites have been previously reported [26]. Like the other two haloviruses, pac occurs within the terS gene, near the stop codon, at a well conserved GC-rich sequence motif. For convenience, base 1 of ChaoS9 was chosen so that it corresponds with the starting bases of phiH1 and phiCh1, which places the pac site terminal base at nt 46 . A summary of the major features of ChaoS9 is given in Table 1, along with the characteristics of phiH1 and phiCh1.

Annotation of the ChaoS9 genome predicted 85 coding sequences (CDS) and one tRNA gene (Figure 5 and Table 2). Most CDS were closely spaced, with 31 overlapping at stop/start codons, and 30 separated by $0-15 \mathrm{nt}$. The majority of CDS were organized into groups having the same orientation, such as $0-25 \mathrm{kbp}$ and $42-55 \mathrm{kbp}$, where all but one CDS are on the upper (forward) strand, and 33.5 to $41.9 \mathrm{kbp}$ where all CDS are on the lower (reverse) strand. The most common stop codon was TGA $(56 ; 65 \%)$, followed by TAA $(19 ; 22 \%)$ and TAG $(10 ; 12 \%)$, a pattern that is similar to the host species, Hbt. salinarum, that also prefers TGA stop codons (TGA, 49\%; TAG, 28\%; TAA, 23\%) (see http:/ / www.kazusa.or.jp/codon).

The ChaoS9 open reading frames (ORFs) were compared (BLASTp) to those from phiH1 and phiCh1 to identify homologs (E-values $\leq 10^{-10}$ ). In some cases, this threshold was relaxed because there was additional support from a conserved gene neighborhood; in other cases, a more stringent threshold was applied in case of casual matches caused by a strong compositional bias. The same process was used to identify homologs in haloarchaeal proviruses (see Section 3.6).

\subsection{Resequencing the Genome of Halovirus PhiCh1}

Given the close similarity of phiCh1 to ChaoS9, it was decided to check the phiCh1 genome sequence by high throughput sequencing using Illumina HiSeq (see methods). This revealed a number of differences to the existing sequence (Genbank: AF440695.1), which are listed in Table S2 along with the genes and proteins affected. Briefly, a total of 40 bases were affected by the revision; 13 point mutations, 9 one-base indels, and one 18 base indel. Overall, the sequence revision made phiCh1 more similar to phiH1 and to ChaoS9. The pac terminal base was determined to be nt $46\left(p=1.23 \times 10^{-23}\right)$, based on analysis of the Illumina reads using the program PhageTerm [38]. This position is consistent with previous studies $[26,43]$.

\subsection{Organisation of the ChaoS9 Genome}

A gene map of ChaoS9 (Figure 5, panel c) is shown between the maps of phiCh1 and phiH1 (panels $b$ and $d)$. Pink shading between the maps indicates regions encoding similar proteins $(\geq 30 \%$ aa identity). Over the first $25 \mathrm{kbp}$, the three viruses share a similar gene synteny, while beyond 25 $\mathrm{kbp}$, ChaoS9 differs considerably from the other two viruses in both gene composition and order. The same pattern is reflected by cumulative AT-skew plots (panel a), which show a similar, steady rise over the first $25 \mathrm{kbp}$ for all three virus genomes, but after this, the plot for ChaoS9 diverges significantly from those of phiCh1 and phiH1. In general, the cumulative AT-skew plots appear to parallel the transcription directions of genes of the three viruses.

The left arm (0-28 kbp) of the ChaoS9 genome. All genes are in the forward direction (top strand) and form a long, functional module specifying proteins putatively involved in DNA packaging, virus structure, and assembly. They include genes encoding the large subunit terminase (TerL) and portal protein (Por), the major virus capsid protein (Mcp), tail sheath, tail-tube, and tape measure (Tmp) proteins, and the tail fiber protein. The gene for the latter protein is also part of an invertible region (see next section). In phiCh1 and phiH1, these genes are expressed during the late phase of lytic infection [44]. The major capsid protein and the tail sheath protein are likely to produce the most prominent bands on SDS-PAGE, which were VP3 and VP4 (Figure 2). The protein molecular weights of Mcp and of the tail sheath protein calculated from their amino acid sequences were $42 \mathrm{kDa}$ (Mcp) and $46.1 \mathrm{kDa}$ (tail sheath), but these values are considerably lower than the observed MWs of VP3 and VP4 (70.2 and $60.2 \mathrm{kDa}$, respectively). After applying the compensatory adjustment for acidic proteins 
reported by Guan et al. [39], the predicted gel sizes of the Mcp (50.4 kDa) and tail sheath protein (56.1 $\mathrm{kDa})$ were still lower than VP3 and VP4. The MW of the tail tube protein $(14.8 \mathrm{kDa})$, after applying the Guan et al. adjustment [39] was predicted to be $19.3 \mathrm{kDa}$ on SDS gels, a value identical to that of VP7. The VP1 band $(143.5 \mathrm{kDa})$ is much larger than any of the annotated virus structural proteins, and may represent a multimeric form.

Sequences of head-neck-tail module proteins can be used to classify caudoviruses [45], and this classified ChaoS9 within the Myoviridae (Type1, Cluster 6). While the gene composition and synteny were well conserved between ChaoS9 and the other viruses, the sequence similarity of genes and proteins revealed major differences between them, suggesting a long history of recombination. For example, the proteins encoded by genes terL to hco of ChaoS9 showed no significant similarity to the corresponding proteins from phiCh1 or phiH1, and this segment includes many of the most highly conserved genes used for virus classification, such as the terminase, portal, and major capsid proteins. The putative assignments of ChaoS9_015 as portal protein and ChaoS9_030 as prohead protease are based on VIRFAM predictions. From 10-25 kbp, the majority of the encoded proteins are related to tail assembly proteins, and most share sequence similarity with the corresponding phiH1 and phiCh1 proteins, with the obvious exceptions (see Figure 5) of the phiCh1 tape measure protein (Tpm) and two hypothetical proteins (PhiH_155, PhiH_160) of phiH1 that immediately precede the tail fiber protein (PhiH_165).

The invertible region (23-29 kbp). The inflection in AT-skew at around $25 \mathrm{kbp}$ occurs at the end of the tail-fiber gene, which is embedded in a segment containing an integrase/recombinase and another tail-fiber related gene (probably a pseudogene) that is inversely oriented to the first one. The similarity of the two fiber genes (pink arrows in Figure 5) can be seen by the crossing of shaded lines (light pink shaded) in this region of Figure 5. Similar gene arrangements to this are found in phiH1 and phiCh1, where it has been shown that the central recombinase allows inversion of the nearby tail-fiber genes, so altering the sequence of the actively expressed copy [46]. The ChaoS9 invertible region contains an ISH12 transposon that is not present in the corresponding invertible regions of the other viruses. This transposon is identical to ISH12 from strain R1 and has targeted the inactive copy of the tail fiber protein (N-term part: ChaoS9_195, C-term part: ChaoS9_160). Curiously, the same transposon is also integrated into phiH1, but at a different genome position.

The right arm (29-55 kbp). This region is the most divergent compared to phiCh1 and phiH1, and contains genes putatively involved in replication ( $\operatorname{par} A, \operatorname{orc} 1$ and $\operatorname{repH}$ ), a tRNA-Arg gene, and numerous genes specifying proteins of unknown function. In both phiH1 and phiCh1, this region has been shown to control lysogeny and the provirus state, maintaining the viral genome as a circular, extrachromasomal dsDNA element [47-49]. Lytic phase gene expression in phiH1 has been shown to be repressed by RepR, a coliphage-like repressor [50-52], but a homologous gene similar to this was not detected in ChaoS9.

There are no DNA methylase genes in ChaoS9, while the other viruses each carry three (e.g., m.I, $\mathrm{m}$.II, and m.III of phiCh1). The corresponding regions of phiH1 and phiCh1 are similar to each other, but contain relatively few genes with matching protein sequences to ChaoS9, and even in these cases the arrangement usually differs. For example, the ISH12 elements of ChaoS9 and phiH1 are $15 \mathrm{kbp}$ apart, in opposite orientation, and in distinct modules. Also, while the RepH proteins show weak similarity to each other, the position of repH in ChaoS9 is about $15 \mathrm{kbp}$ further right compared to the repH genes of phiCh1 and phiH1. Even the type of replication related genes differs, with ChaoS9 carrying a gene similar to Orc (orc1) that is not present in the other viruses, while phiH1 and phiCh1 carry a gene similar to PCNA (pcnA) that is not found in ChaoS9. In phiH1, the L-region has been shown to be able to replicate independently as a plasmid [41], and also contains an immunity gene (imm) near repH that protects L-plasmid containing host cells from lytic infection by phiH1 [41]. The same arrangement is found in phCh1. While a gene related to imm was not detected near repH of ChaoS9, a gene specifying a MarR-like repressor (ChaoS9_390) is present just downstream of repH. 
ChaoS9 is the only virus of the three predicted to carry a tRNA gene, tRNA-Arg(TCG). In BLASTn searches, this sequence is unlike other tRNAs except for a conserved region of the right arm (nt 43-70), which matches many haloarchaeal and bacterial tRNAs. Curiously, the best matches are to cyanobacteria tRNAs, such as tRNA Gly (CCC) of Synechococcus sp. KORDI-100 (CP006269 nt 191738-191667), which gives a $28 \mathrm{nt}$ perfect alignment. In haloarchaea, the best match found was $19 \mathrm{nt}$. Although the ChaoS9 tRNA appears to be complete, its function is less clear. Ostensibly, it specifies arginine (anticodon TCG), and the corresponding codon is the third most frequent Arg codon used in both the host species Hbt. salinarum [53] and ChaoS9, but there is no large difference in usage between the two (7\% in Halobacterium and $11 \%$ in ChaoS9), and there are much rarer codons for this amino acid used by both host and virus. It could also represent an att sequence used for integration of the viral genome into tRNA genes of host strains (see later) or may have a regulatory role.

\subsection{Related Provirus-Like Matches in Haloarchaea}

Several haloarchaeal genomes carried provirus elements related to ChaoS9, and the gene maps of two examples (PVH3A1 and PVHS1) are depicted in Figure 6. The attL and attR sites of both proviruses indicate they were integrated into tRNA genes (tRNA-Met and tRNA-Cys), probably mediated by the integrases encoded at their right ends. Both appear to be intact and probably functional, as their left halves possess complete suites of virus structural and assembly genes. Their right halves predominantly carry genes for uncharacterized proteins unrelated to ChaoS9. A few genes in this half, and at the extreme left end (before the structural/assembly genes), could be assigned functions, such as the replication protein Orc, and DNA methylases (Mtase, Dam). Neither provirus carried a region corresponding to the invertible tail-fiber genes of ChaoS9.

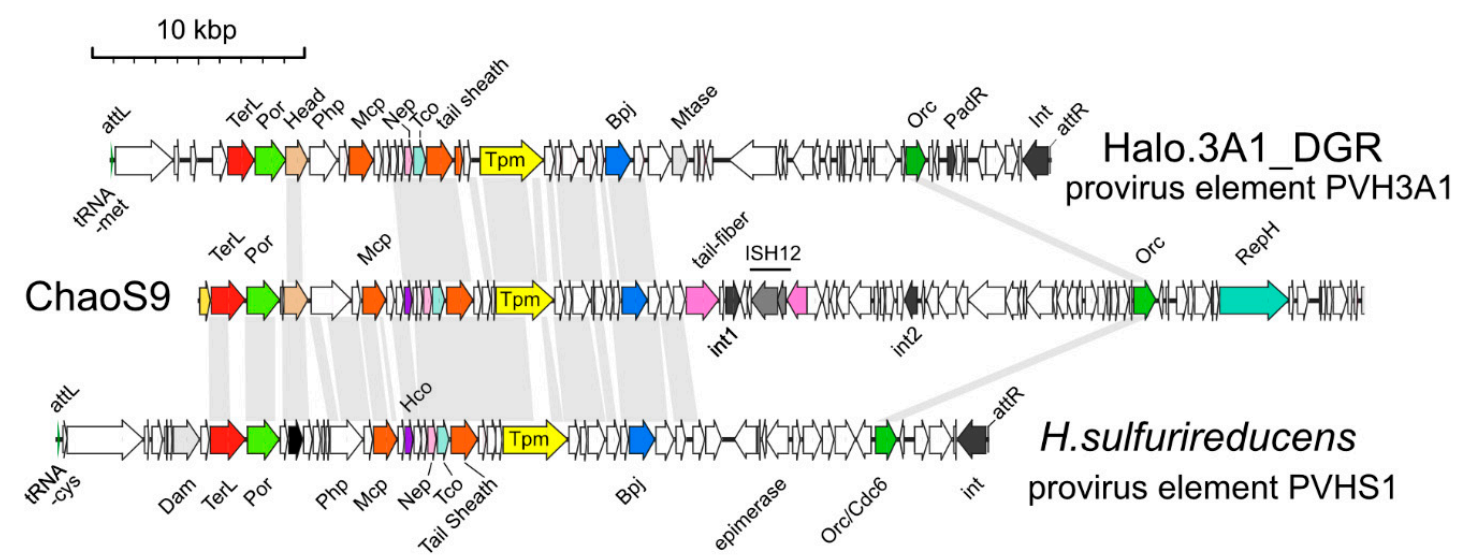

Figure 6. Provirus elements PVH3A1 and PVHS1 compared to ChaoS9. PVH3A1 from haloarchaeon strain 3A1_DGR (accession NZ_KK033114; nt 1475908-1520418), and PVHS1 from Haa. sulfurireducens strain M27-SA2 (accession NZ_CP011564; nt 1971038- 1927125), are compared to ChaoS9. Grey bands connecting genome maps represent similarity (tBLASTx, $>30 \%$ identity) between the inferred proteins of ChaoS9 and each provirus. Positions of several annotated proteins and their genes are indicated by color and name; TerL, large subunit terminase (red); Por, portal protein (light green); Head, SPP1_gp7 family head assembly protein, (light brown); capsid proteins such as Mcp (major capsid protein) and Tail sheath protein (brown); Tpm, tape measure protein (yellow); Dam, adenine methyltransferase (light grey); Bpj, baseplate J family protein (blue); Orc1, replication protein Orc1 (green); RepH, plasmid replication protein (turquoise). Scale bar, in kbp, is shown at top.

The right arm (29-55 kbp) of ChaoS9 shows little similarity to either provirus except for Orc1. Across the left arm (0-28 kbp), the region covering virus structural and assembly proteins, ChaoS9 maintains good synteny and protein similarity to PVHS1, but with PVH3A1 there is a distinct break in similarity within the virus structural/assembly gene module. PVH3A1 neck and tail proteins are similar to ChaoS9 but the head/assembly proteins are unrelated, except for the head assembly 
protein (labeled Head; Figure 6, panel a). PhiH1, and to a lesser extent phiCh1, show a comparable break in similarity to ChaoS9 head and tail genes (Figure 5). All of these examples suggest that ancestral recombination events between ChaoS9-like viruses have occurred within genes located between the head morphogenesis genes and the tail morphogenesis genes, and that these often result in viable progeny.

The distinct right halves of the proviruses suggest that they have also undergone extensive recombination relative to ChaoS9, perhaps reflecting differences in virulence and/or provirus state.

\subsection{A Diverse Family of Haloviruses}

In order to examine evolutionary relationships between ChaoS9 and other viruses and proviruses, genes were sought that were both sufficiently conserved and present in all examples. As shown in Figures 5 and 6, relatively few genes matched these criteria. Even the large subunit terminase (TerL) and major capsid protein (Mcp) were poorly conserved, and these have been widely used in previous studies of bacteriophages for delineating virus taxa [4]. The Bpj (baseplate J family protein) and the tail sheath proteins were selected to infer phylogenetic relationships as they were relatively long, conserved in sequence and present in all examples. BLASTp searches (accessed 20 November 2018) with ChaoS9 Bpj retrieved over 50 high scoring matches (E value $<10^{-30}$ ), with the closest matches all being from haloarchaea or haloviruses, while less similar matches included proteins from Bacteria and bacteriophages. An inferred phylogenetic tree based on alignment of Bpj (Figure 7a) shows that ChaoS9 is part of a robust clade (100\% bootstrap confidence) which includes haloviruses phiCh1, phiH1, and provirus-like elements of four haloarchaea belonging to at least three different genera. A separate clade, also with high bootstrap confidence, contains four haloviruses (HF1, HF2, HRTV-8, and HSTV-2) and a provirus element of Haloferax larsenii.

BLASTp searches of the NCBI database with the ChaoS9 tail sheath protein (ChaoS9_080) retrieved only nine high scoring matches (E value $<10^{-35}$ ), and these were all from haloarchaea or haloviruses present in the Bpj tree, and most closely related to ChaoS9. The inferred phylogenetic tree based on this protein (Figure $7 \mathrm{~b}$ ) reveals a topology similar to that of Bpj proteins.

The major capsid protein (Mcp) of ChaoS9 was used to search the NCBI database (BLASTp, nr database, accessed 22 November 2018) and retrieved only five matches (Figure 7c), which varied in similarity from 36 to $76 \%$ (aa identity). Three were from organisms previously identified as specifying ChaoS9-related proteins (Haa. sulfurireducens, Saliphagus sp. LR7 and Hpt. malekzadehii) and are present in Figures 6 and 7, while the other two sequences were from Salinigranum rubrum and the tailed halovirus HHTV-1 [5,54].

The DNA sequences of HHTV-1 and ChaoS9 share no significant similarity (Figure 4), but a BLASTp comparison of all ChaoS9 and HHTV-1 proteins found that Mcp was the only protein with significant similarity ( $36 \%$ ) shared between these haloviruses. Such a pattern of similarity between tailed viruses that is strictly limited to the Mcp appears to be uncommon in the published literature. A less clear-cut but comparable example occurs between actinophages Jace and Tweety (accessions EF536069 and MH153804), which share similar Mcp (32\%) and integrase (39\%) protein sequences but weak or negligible similarity between all other proteins. Cases where head genes and tail genes derive from different virus lineages are slightly more common [55,56] (see Discussion). 


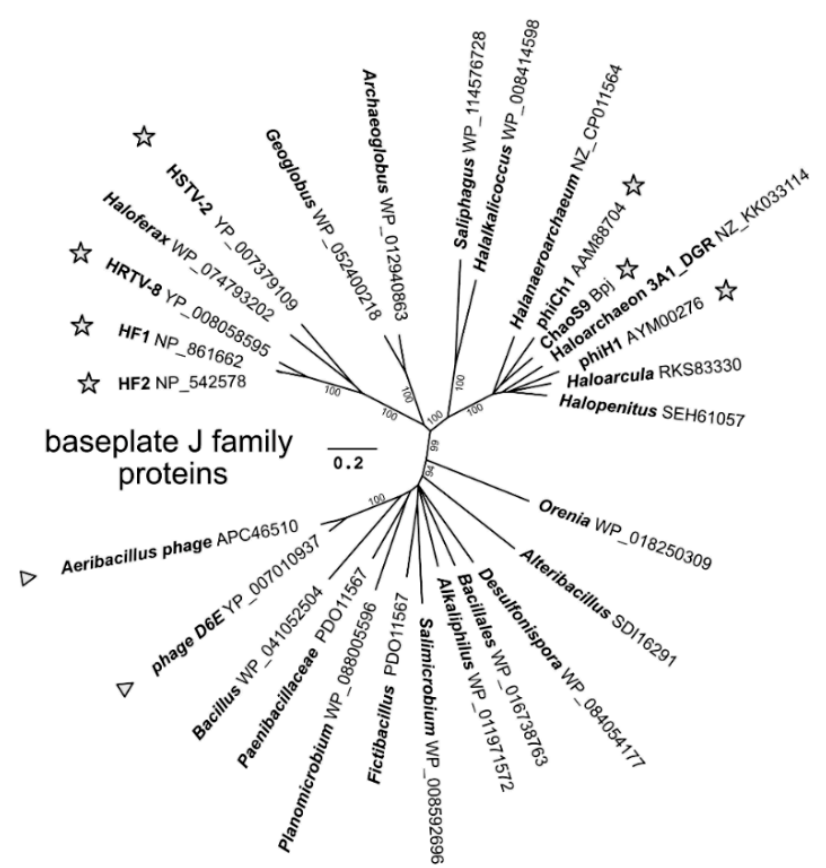

(a)

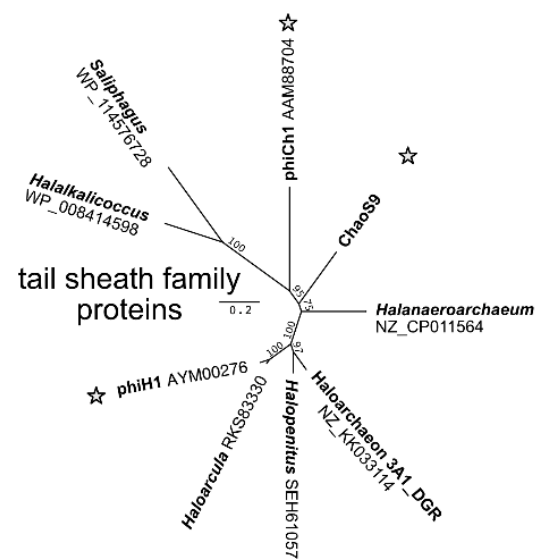

(b)

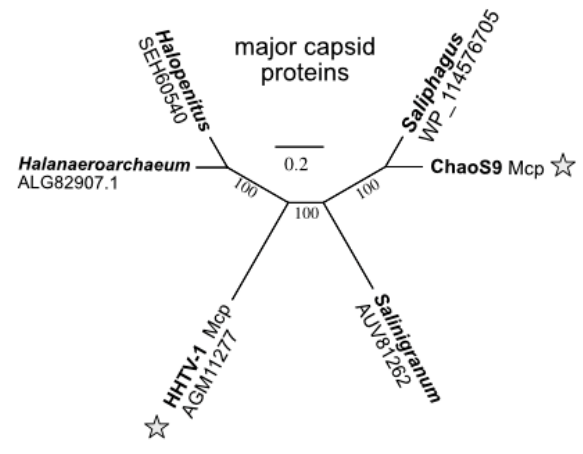

(c)

Figure 7. Phylogenetic tree reconstructions using (a) baseplate J family proteins, (b) major tail sheath proteins and (c), major capsid proteins. Halovirus proteins are marked by stars and bacteriophage proteins are marked by triangles. The consensus trees were produced using the Neighbor-Joining algorithm and 100 bootstrap replications. Scale bars represent the estimated number of amino acid replacements per position.

\subsection{CRISPR Spacer Matches to ChaoS9}

The ChaoS9 genome was used to search for matching CRISPR spacer sequences available from public databases (see methods). A total of 277 spacers were identified that closely matched ChaoS9, with the majority of spacers originating from Antarctic lake metagenomes (Deep Lake, Rauer Lake and Club Lake). After removing duplicates, the number of distinct spacers was reduced to 39, and in Table S3 the matching spacers have been ordered by their position along the ChaoS9 genome. The distribution of spacers is highly skewed, with most (34/39) targeting sequences within the right arm, and particularly the repH gene, for which there were 14 distinct spacer sequences. The direct repeats (DR) of the matching spacers were most similar or identical to those found in CRISPR arrays of sequenced haloarchaea, particularly antarctic isolates such as Halorubrum lacusprofundi, Halobacterium sp. DL1 and haloarchaeon DL3. For comparison, the phiH1 and phiCh1 genomes were also scanned for matching spacers in the same antarctic lake metagenomes (IMG/VR webserver; 
(https://img.jgi.doe.gov/cgi-bin/vr/main.cgi; accessed 20 December 2018). This returned only 2 (phiCh1) and 3 (phiH1) significant matches (supplementary Table S4), of which all of the phiH1 matching spacers were to the tail fiber gene (PhiH1_165), as was one of the spacers matching phiCh1 (PhiCh1_155). The remaining spacer to phiCh1 matched a sequence within the prohead protease gene (gpB, PhiCh1_045). All six spacers were from Deep Lake and Rauer Lake metagenomes.

\section{Discussion}

This study focused on identifying the cause of a lysis event in a large, laboratory culture of Hbt. salinarum S9. A novel myovirus was recovered, ChaoS9, with morphological and molecular characteristics specifically resembling myoviruses phiH and phiCh1, but which differed significantly in sequence from both of these. An earlier lysis event in the same laboratory that affected a culture of Hbt. salinarum R1 was shown to have been caused by phiH, however, the 2007 event was not a recurrent infection. The sources of both infections are unknown, possibly raw salt, but these events highlight the need for preventative measures, even though the high salt conditions used for cultivation of haloarchaea are generally regarded as providing a strong barrier to contamination by non-halophilic microorganisms. However, tailed viruses (caudoviruses) such as ChaoS9 are not only the most common type of prokaryotic virus, but together with other bacterial and archaeal viruses, they represent the most abundant biological entity on Earth, estimated to be $10^{31}$ virions [57]. It is not surprising, then, that virus contamination and lysis events are a constant threat in large-scale commercial fermentations [22], and cause such significant losses that systematic preventative programs have been formulated, such as PACCP (phage analysis and critical control point) [58].

ChaoS9 was most similar in morphology, genome type, genome length, and sequence to the tailed haloviruses phiH1, and phiCh1 [26,43]. The dsDNA genome was terminally redundant and circularly permuted, consistent with headful packaging, as has been shown for the related viruses. The likely pac site was identified by sequence homology. Gene synteny of the virus morphogenesis genes of the left arm of the genome was similar to those of phiH1 and phiCh1, while the right arm comprised genes for DNA replication, plasmid partition, and a tRNA, as well as many genes specifying proteins of unknown function. The right arm corresponds to the lysogeny, replication, and accessory gene region of phiH1 and phiCh1, and probably serves the same general function in ChaoS9. A characteristic feature of phiH1 and phiCh1 is an invertible tail fiber gene module, which was also present in ChaoS9, except that it also included an ISH12 transposon.

Comparison of the ChaoS9 genome with those of phiH1 and phiCh1 revealed a distinctive pattern of similarity and difference, suggesting an evolutionary history involving large recombination events. While the tail gene region of ChaoS9 was similar to the other two viruses, the head/assembly genes of the left arm of the genome, as well as most of right arm (the replication/lysogeny region), were not. This means that the major capsid protein, terminase (large subunit) and portal proteins of ChaoS9 are all unrelated to those of phiH1 and phiCh1. The most parsimonious explanation is that a recombination event has replaced the head morphogenesis module while leaving the tail morphogenesis module intact. It appears to be a chimeric recombinant, with the point of similarity disjuncture occurring between the putative head closure (hco, ChaoS9_055) and neck protein (nep, ChaoS9_070) genes. Similar examples where head morphogenesis and tail morphogenesis modules appear to derive from different virus types are not uncommon in the literature, such as the Gordonia spp. phage Kita (Figure 4 of [55]), and Xylella fastidiosa phage Xfas53 [56], phi80 and Gifsy-2 [59]. The likelihood of such recombination events producing viable progeny is increased because of the way caudoviruses are assembled, with heads and tails produced as separate structures that are then joined together [60].

Provirus elements related to ChaoS9 were found among haloarchaeal genome sequences, integrated into their host chromosomes via tRNA genes, which supports the view that ChaoS9, like phiH1 and phiCh1, has a temperate lifestyle [61]. One provirus (PVHS1) showed good sequence similarity to ChaoS9 proteins across most of the virus morphogenesis genes, while a second provirus (PVH3A1) showed a general pattern of sequence similarity to ChaoS9 like that of phiH1 and phiCh1; 
with mainly unrelated head morphogenesis genes (including dissimilar major capsid proteins), similar tail morphogenesis genes, and largely unrelated right arms (lysogeny/replication). The right arms of both proviruses differed significantly from the right arm of the ChaoS9 genome, but all carried a putative orc1 gene, presumably for use in replication as a (provirus) plasmid if the circularized genome is unable to integrate into the host chromosome via attP. These two examples again point to frequent recombination events that exchange the head morphogenesis module while retaining the tail morphogenesis genes.

Curiously, the ChaoS9 major capsid protein is most similar to that of the haloarchaeal siphovirus HHTV-1, but this is the only protein between them that is similar, and they are otherwise unrelated. In studies of enterobacterial viruses, major capsid protein (MCP) gene exchange was estimated to be extremely rare between phage clusters or types [61]. How this occurred in the evolutionary history of these viruses will be intriguing to resolve, but presumably indicates widespread recombination between tailed viruses of haloarchaea.

A surprisingly large number of identical or near identical CRISPR spacers to ChaoS9 were detected, and even more surprising was their identification in the metagenomes from Antarctic salt lakes. The majority of target sites were located in the right arm of the genome, in the lysogeny/replication region. A few were to sites within the left arm of the genome, such as the tail fiber gene. In contrast to ChaoS9, the genomes of phiH1 and phiCh1 matched only a few spacers from the same Antarctic metagenomes, and all but one targeted the tail fiber gene. This suggests that these lakes harbor tailed viruses with a lysogeny/replication module, similar to ChaoS9. Their tail fiber genes also show significant sequence similarity to ChaoS9, phiH1, and phiCh1, but the other virus morphogenesis genes are distinct.

The precise relationship between ChaoS9, phiCh1, and phiH1 was explored in several ways. They share average nucleotide identity (ANIb) values of $\geq 74 \%$, and phylogenetic tree reconstructions using baseplate J virus protein sequences clustered ChaoS9, phiH1, and phiCh1 into a well-supported clade, distinct from other tailed haloviruses. The close relationship is consistent with the other comparative data (morphology, genome size and packaging, gene synteny, and inferred protein sequences). Halobacterium virus phiH is the type species of the genus Myohalovirus and a previous study has shown that phiCh1 should be included in the same genus, as their genomes share $63 \%$ nucleotide identity, are mostly colinear, and their proteins show, on average, 70\% amino acid identity [26]. PhiCh1 proteins affected by genome revision became more similar to those of phiH1. ChaoS9 shows many similarities and correspondences to phiH1 and phiCh1 but also considerable differences, including a distinct major capsid protein, terminase (large subunit) and portal protein, and is certainly a distinct species to phiH1 and phiCh1, but whether it should be classified outside of the Myohalovirus genus requires further consideration.

Supplementary Materials: The following are available online at http:/ / www.mdpi.com/2073-4425/10/3/194/s1, Figure S1: Experimental and in silico restriction digests of ChaoS9 DNA. Table S1; DNA primers used for PCR or sequencing viral DNA. Table S2; Revisions of phiCh1 genes after re-sequencing the genome. Table S3; CRISPR spacers matching ChaoS9; Table S4, CRISPR spacers matching phiH1 and phiCh1.

Author Contributions: Conceptualization, visualization, investigation, M.D.-S.; investigation (electron microscopy) G.W.; investigation (genome sequencing) P.P.; data curation, F.P., M.D.-S.; funding acquisition, D.O.; project administration, F.P.; resources, A.W.; writing—original draft, F.P., M.D.-S.; writing—review \& editing, A.W., D.O., F.P., M.D.-S.; validation, A.W.

Funding: This research received no external funding.

Acknowledgments: We wish to thank Cyril Boulegue, Beatrix Scheffer, and Snezan Marinkovic (core facility, MPI) for their contribution to genome sequencing. We thank Graham Hatfull for advice on bacteriophage diversity.

Conflicts of Interest: The authors declare no conflict of interest. 


\section{References}

1. Dyall-Smith, M.; Tang, S.L.; Bath, C. Haloarchaeal viruses: How diverse are they? Res. Microbiol. 2003, 154, 309-313. [CrossRef]

2. Krupovic, M.; Cvirkaite-Krupovic, V.; Iranzo, J.; Prangishvili, D.; Koonin, E.V. Viruses of archaea: Structural, functional, environmental and evolutionary genomics. Virus Res. 2017, 244, 181-193. [CrossRef] [PubMed]

3. Oren, A.; Bratbak, G.; Heldal, M. Occurrence of virus-like particles in the Dead Sea. Extremophiles 1997, 1, 143-149. [CrossRef] [PubMed]

4. Krupovic, M.; Forterre, P.; Bamford, D.H. Comparative analysis of the mosaic genomes of tailed archaeal viruses and proviruses suggests common themes for virion architecture and assembly with tailed viruses of bacteria. J. Mol. Biol. 2010, 397, 144-160. [CrossRef] [PubMed]

5. Sencilo, A.; Jacobs-Sera, D.; Russell, D.A.; Ko, C.C.; Bowman, C.A.; Atanasova, N.S.; Osterlund, E.; Oksanen, H.M.; Bamford, D.H.; Hatfull, G.F.; et al. Snapshot of haloarchaeal tailed virus genomes. RNA Biol. 2013, 10, 803-816. [CrossRef] [PubMed]

6. Sencilo, A.; Roine, E. A glimpse of the genomic diversity of haloarchaeal tailed viruses. Front. Microbiol. 2014, 5, 84. [PubMed]

7. Atanasova, N.S.; Bamford, D.H.; Oksanen, H.M. Virus-host interplay in high salt environments. Environ. Microbiol. Rep. 2016, 8, 431-444. [CrossRef] [PubMed]

8. Zhang, Z.; Liu, Y.; Wang, S.; Yang, D.; Cheng, Y.; Hu, J.; Chen, J.; Mei, Y.; Shen, P.; Bamford, D.H.; et al. Temperate membrane-containing halophilic archaeal virus SNJ1 has a circular dsDNA genome identical to that of plasmid pHH205. Virology 2012, 434, 233-241. [CrossRef] [PubMed]

9. Liu, Y.; Wang, J.; Liu, Y.; Wang, Y.; Zhang, Z.; Oksanen, H.M.; Bamford, D.H.; Chen, X. Identification and characterization of SNJ2, the first temperate pleolipovirus integrating into the genome of the SNJ1-lysogenic archaeal strain. Mol. Microbiol. 2015, 98, 1002-1020. [CrossRef] [PubMed]

10. Demina, T.A.; Pietila, M.K.; Svirskaite, J.; Ravantti, J.J.; Atanasova, N.S.; Bamford, D.H.; Oksanen, H.M. Archaeal Haloarcula californiae Icosahedral virus 1 highlights conserved elements in icosahedral membrane-containing DNA viruses from extreme environments. MBio 2016, 7, e00699-16. [CrossRef] [PubMed]

11. Hartmann, R.; Oesterhelt, D. Bacteriorhodopsin-mediated photophosphorylation in Halobacterium halobium. Eur. J. Biochem. 1977, 77, 325-335. [CrossRef] [PubMed]

12. Michel, H.; Oesterhelt, D. Electrochemical proton gradient across the cell membrane of Halobacterium halobium: Effect of N,N'-dicyclohexylcarbodiimide, relation to intracellular adenosine triphosphate, adenosine diphosphate, and phosphate concentration, and influence of the potassium gradient. Biochemistry 1980, 19, 4607-4614. [PubMed]

13. Haupts, U.; Tittor, J.; Oesterhelt, D. Closing in on bacteriorhodopsin: Progress in understanding the molecule. Annu. Rev. Biophys. Biomol. Struct. 1999, 28, 367-399. [CrossRef] [PubMed]

14. Mirfeizollahi, A.; Yakhchali, B.; Deldar, A.A.; Karkhane, A.A. In silico and experimental improvement of bacteriorhodopsin production in Halobacterium salinarum R1 by increasing DNA-binding affinity of Bat through Q661R/Q665R substitutions in HTH motif. Extremophiles 2019, 23, 59-67. [CrossRef] [PubMed]

15. Stoeckenius, W.; Lozier, R.H.; Bogomolni, R.A. Bacteriorhodopsin and the purple membrane of halobacteria. Biochim. Biophys. Acta (BBA)—Rev. Bioenerg. 1979, 505, 215-278. [CrossRef]

16. Baliga, N.S.; DasSarma, S. Saturation mutagenesis of the TATA box and upstream activator sequence in the haloarchaeal bop gene promoter. J. Bacteriol. 1999, 181, 2513-2518. [PubMed]

17. Baliga, N.S.; Kennedy, S.P.; Ng, W.V.; Hood, L.; DasSarma, S. Genomic and genetic dissection of an archaeal regulon. Proc. Natl. Acad. Sci. USA 2001, 98, 2521-2525. [CrossRef] [PubMed]

18. Baliga, N.S.; Pan, M.; Goo, Y.A.; Yi, E.C.; Goodlett, D.R.; Dimitrov, K.; Shannon, P.; Aebersold, R.; Ng, W.V.; Hood, L. Coordinate regulation of energy transduction modules in Halobacterium sp. analyzed by a global systems approach. Proc. Natl. Acad. Sci. USA 2002, 99, 14913-14918. [CrossRef] [PubMed]

19. Yang, C.F.; Kim, J.M.; Molinari, E.; DasSarma, S. Genetic and topological analyses of the bop promoter of Halobacterium halobium: Stimulation by DNA supercoiling and non-B-DNA structure. J. Bacteriol. 1996, 178, 840-845. [CrossRef] [PubMed]

20. Rudolph, J.; Nordmann, B.; Storch, K.F.; Gruenberg, H.; Rodewald, K.; Oesterhelt, D. A family of halobacterial transducer proteins. FEMS Microbiol. Lett. 1996, 139, 161-168. [CrossRef] [PubMed] 
21. Storch, K.F.; Rudolph, J.; Oesterhelt, D. Car: A cytoplasmic sensor responsible for arginine chemotaxis in the archaeon Halobacterium salinarum. EMBO J. 1999, 18, 1146-1158. [CrossRef] [PubMed]

22. Marco, M.B.; Moineau, S.; Quiberoni, A. Bacteriophages and dairy fermentations. Bacteriophage 2012, 2, 149-158. [CrossRef] [PubMed]

23. Schnabel, H.; Zillig, W.; Pfaffle, M.; Schnabel, R.; Michel, H.; Delius, H. Halobacterium halobium phage $\Phi H$. EMBO J. 1982, 1, 87-92. [CrossRef] [PubMed]

24. Soppa, J.; Oesterhelt, D. Bacteriorhodopsin mutants of Halobacterium sp. GRB. I. The 5-bromo-2'-deoxyuridine selection as a method to isolate point mutants in halobacteria. J. Biol. Chem. 1989, 264, 13043-13048. [PubMed]

25. Gordon, D. Viewing and editing assembled sequences using Consed. Curr. Protoc. Bioinform. 2003, 2, 11.2.1-11.2.43.

26. Dyall-Smith, M.; Pfeifer, F.; Witte, A.; Oesterhelt, D.; Pfeiffer, F. Complete genome sequence of the model halovirus phiH1 (ФH1). Genes 2018, 9, 493. [CrossRef] [PubMed]

27. Lomsadze, A.; Gemayel, K.; Tang, S.; Borodovsky, M. Modeling leaderless transcription and atypical genes results in more accurate gene prediction in prokaryotes. Genome Res. 2018, 28, 1079-1089. [CrossRef] [PubMed]

28. Siddaramappa, S.; Challacombe, J.F.; Decastro, R.E.; Pfeiffer, F.; Sastre, D.E.; Gimenez, M.I.; Paggi, R.A.; Detter, J.C.; Davenport, K.W.; Goodwin, L.A.; et al. A comparative genomics perspective on the genetic content of the alkaliphilic haloarchaeon Natrialba magadii ATCC 43099 ${ }^{\mathrm{T}}$. BMC Genom. 2012, 13, 165. [CrossRef] [PubMed]

29. Laemmli, U.K. Cleavage of structural proteins during the assembly of the head of the bacteriophage T4. Nature 1970, 227, 680-685. [CrossRef] [PubMed]

30. Kearse, M.; Moir, R.; Wilson, A.; Stones-Havas, S.; Cheung, M.; Sturrock, S.; Buxton, S.; Cooper, A.; Markowitz, S.; Duran, C.; et al. Geneious Basic: An integrated and extendable desktop software platform for the organization and analysis of sequence data. Bioinformatics 2012, 28, 1647-1649. [CrossRef] [PubMed]

31. Lopes, A.; Tavares, P.; Petit, M.A.; Guerois, R.; Zinn-Justin, S. Automated classification of tailed bacteriophages according to their neck organization. BMC Genom. 2014, 15, 1027. [CrossRef] [PubMed]

32. Ovcharenko, I.; Loots, G.G.; Hardison, R.C.; Miller, W.; Stubbs, L. zPicture: Dynamic alignment and visualization tool for analyzing conservation profiles. Genome Res. 2004, 14, 472-477. [CrossRef] [PubMed]

33. Yoon, S.H.; Ha, S.M.; Lim, J.; Kwon, S.; Chun, J. A large-scale evaluation of algorithms to calculate average nucleotide identity. Antonie Van Leeuwenhoek 2017, 110, 1281-1286. [CrossRef] [PubMed]

34. Babicki, S.; Arndt, D.; Marcu, A.; Liang, Y.; Grant, J.R.; Maciejewski, A.; Wishart, D.S. Heatmapper: Web-enabled heat mapping for all. Nucleic Acids Res. 2016, 44, W147-W153. [CrossRef] [PubMed]

35. Grissa, I.; Vergnaud, G.; Pourcel, C. CRISPRcompar: A website to compare clustered regularly interspaced short palindromic repeats. Nucleic Acids Res. 2008, 36, W145-W148. [CrossRef] [PubMed]

36. Paez-Espino, D.; Chen, I.A.; Palaniappan, K.; Ratner, A.; Chu, K.; Szeto, E.; Pillay, M.; Huang, J.; Markowitz, V.M.; Nielsen, T.; et al. IMG/VR: A database of cultured and uncultured DNA Viruses and retroviruses. Nucleic Acids Res. 2017, 45, D457-D465. [CrossRef] [PubMed]

37. Skennerton, C.T.; Imelfort, M.; Tyson, G.W. Crass: Identification and reconstruction of CRISPR from unassembled metagenomic data. Nucleic Acids Res. 2013, 41, e105. [CrossRef] [PubMed]

38. Garneau, J.R.; Depardieu, F.; Fortier, L.C.; Bikard, D.; Monot, M. PhageTerm: A tool for fast and accurate determination of phage termini and packaging mechanism using next-generation sequencing data. Sci. Rep. 2017, 7, 8292. [CrossRef] [PubMed]

39. Guan, Y.; Zhu, Q.; Huang, D.; Zhao, S.; Jan Lo, L.; Peng, J. An equation to estimate the difference between theoretically predicted and SDS PAGE-displayed molecular weights for an acidic peptide. Sci. Rep. 2015, 5, 13370. [CrossRef] [PubMed]

40. ICTV Report. ICTV Online (10th) Report on Virus Taxonomy. Available online: https:/ /talk.ictvonline.org/ taxonomy/p/taxonomy-history?taxnode_id=20170459 (accessed on 19 March 2018).

41. Schnabel, H. An immune strain of Halobacterium halobium carries the invertible L segment of phage $\Phi \mathrm{H}$ as a plasmid. Proc. Natl. Acad. Sci. USA 1984, 81, 1017-1020. [CrossRef] [PubMed]

42. Witte, A.; Baranyi, U.; Klein, R.; Sulzner, M.; Luo, C.; Wanner, G.; Krüger, D.H.; Lubitz, W. Characterization of Natronobacterium magadii phage $\varphi \mathrm{Ch1}$, a unique archaeal phage containing DNA and RNA. Mol. Microbiol. 1997, 23, 603-616. [CrossRef] [PubMed] 
43. Klein, R.; Baranyi, U.; Rössler, N.; Greineder, B.; Scholz, H.; Witte, A. Natrialba magadii virus $\varphi$ Ch1: First complete nucleotide sequence and functional organization of a virus infecting a haloalkaliphilic archaeon. Mol. Microbiol. 2002, 45, 851-863. [CrossRef] [PubMed]

44. Stolt, P.; Zillig, W. Gene regulation in halophage $\Phi H$; more than promoters. Syst. Appl. Microbiol. 1993, 16, 591-596. [CrossRef]

45. VIRFAM. Remote Homology Detection of Viral Protein Families. Available online: http://biodev.cea.fr/ virfam/ (accessed on 8 October 2018).

46. Klein, R.; Rossler, N.; Iro, M.; Scholz, H.; Witte, A. Haloarchaeal myovirus phiCh1 harbours a phase variation system for the production of protein variants with distinct cell surface adhesion specificities. Mol. Microbiol. 2012, 83, 137-150. [CrossRef] [PubMed]

47. Schnabel, H.; Zillig, W. Circular structure of the genome of phage $\Phi H$ in a lysogenic Halobacterium halobium. Mol. Gen. Genet. 1984, 193, 422-426. [CrossRef]

48. Iro, M.; Klein, R.; Galos, B.; Baranyi, U.; Rossler, N.; Witte, A. The lysogenic region of virus phiCh1: Identification of a repressor-operator system and determination of its activity in halophilic Archaea. Extremophiles 2007, 11, 383-396. [CrossRef] [PubMed]

49. Gropp, F.; Grampp, B.; Stolt, P.; Palm, P.; Zillig, W. The immunity-conferring plasmid p $\varphi$ HL from the Halobacterium salinarium phage $\varphi \mathrm{H}$ : Nucleotide sequence and transcription. Virology 1992, 190, 45-54. [CrossRef]

50. Ken, R.; Hackett, N.R. Halobacterium halobium strains lysogenic for phage phiH contain a protein resembling coliphage repressors. J. Bacteriol. 1991, 173, 955-960. [CrossRef] [PubMed]

51. Stolt, P.; Zillig, W. In vivo studies on the effects of immunity genes on early lytic transcription in the Halobacterium salinarium phage $\varphi$ H. Mol. Gen. Genet. 1992, 235, 197-204. [CrossRef] [PubMed]

52. Stolt, P.; Zillig, W. Transcription of the halophage $\Phi H$ repressor gene is abolished by transcription from an inversely oriented lytic promoter. FEBS Lett. 1994, 344, 125-128. [CrossRef]

53. Pfeiffer, F.; Schuster, S.C.; Broicher, A.; Falb, M.; Palm, P.; Rodewald, K.; Ruepp, A.; Soppa, J.; Tittor, J.; Oesterhelt, D. Evolution in the laboratory: The genome of Halobacterium salinarum strain R1 compared to that of strain NRC-1. Genomics 2008, 91, 335-346. [CrossRef] [PubMed]

54. Svirskaite, J.; Oksanen, H.M.; Daugelavicius, R.; Bamford, D.H. Monitoring physiological changes in haloarchaeal cell during virus release. Viruses 2016, 8, 59. [CrossRef] [PubMed]

55. Pope, W.H.; Mavrich, T.N.; Garlena, R.A.; Guerrero-Bustamante, C.A.; Jacobs-Sera, D.; Montgomery, M.T.; Russell, D.A.; Warner, M.H.; Science Education Alliance-Phage Hunters Advancing, G.; Evolutionary, S.; et al. Bacteriophages of Gordonia spp. display a spectrum of diversity and genetic relationships. MBio 2017, 8, e01069-17. [CrossRef] [PubMed]

56. Summer, E.J.; Enderle, C.J.; Ahern, S.J.; Gill, J.J.; Torres, C.P.; Appel, D.N.; Black, M.C.; Young, R.; Gonzalez, C.F. Genomic and biological analysis of phage Xfas53 and related prophages of Xylella fastidiosa. J. Bacteriol. 2010, 192, 179-190. [CrossRef] [PubMed]

57. Hendrix, R.W.; Smith, M.C.; Burns, R.N.; Ford, M.E.; Hatfull, G.F. Evolutionary relationships among diverse bacteriophages and prophages: All the world's a phage. Proc. Natl. Acad. Sci. USA 1999, 96, 2192-2197. [CrossRef] [PubMed]

58. Samson, J.E.; Moineau, S. Bacteriophages in food fermentations: New frontiers in a continuous arms race. Annu. Rev. Food Sci. Technol. 2013, 4, 347-368. [CrossRef] [PubMed]

59. Grose, J.H.; Casjens, S.R. Understanding the enormous diversity of bacteriophages: The tailed phages that infect the bacterial family Enterobacteriaceae. Virology 2014, 468-470, 421-443. [CrossRef] [PubMed]

60. Fokine, A.; Rossmann, M.G. Common evolutionary origin of procapsid proteases, phage tail tubes, and tubes of bacterial type VI secretion systems. Structure 2016, 24, 1928-1935. [CrossRef] [PubMed]

61. Casjens, S.R.; Grose, J.H. Contributions of P2- and P22-like prophages to understanding the enormous diversity and abundance of tailed bacteriophages. Virology 2016, 496, 255-276. [CrossRef] [PubMed]

(C) 2019 by the authors. Licensee MDPI, Basel, Switzerland. This article is an open access article distributed under the terms and conditions of the Creative Commons Attribution (CC BY) license (http://creativecommons.org/licenses/by/4.0/). 